\title{
Diffusion with resetting in a logarithmic potential
}

\author{
Somrita Ray ${ }^{1, a)}$ and Shlomi Reuveni ${ }^{1, b)}$ \\ School of Chemistry, The Center for Physics and Chemistry of Living Systems, The Raymond and Beverly Sackler Center for \\ Computational Molecular and Materials Science, \& The Ratner Center for Single Molecule Science, Tel Aviv University, \\ Tel Aviv 69978, Israel
}

(Dated: 14 April 2020)

\begin{abstract}
We study the effect of resetting on diffusion in a logarithmic potential. In this model, a particle diffusing in a potential $U(x)=U_{0} \log |x|$ is reset, i.e., taken back to its initial position, with a constant rate $r$. We show that this analytically tractable model system exhibits a series of phase transitions as a function of a single parameter, $\beta U_{0}$, the ratio of the strength of the potential to the thermal energy. For $\beta U_{0}<-1$ the potential is strongly repulsive, preventing the particle from reaching the origin. Resetting then generates a non-equilibrium steady state which is characterized exactly and thoroughly analyzed. In contrast, for $\beta U_{0}>-1$ the potential is either weakly repulsive or attractive and the diffusing particle eventually reaches the origin. In this case, we provide a closed form expression for the subsequent first-passage time distribution and show that a resetting transition occurs at $\beta U_{0}=5$. Namely, we find that resetting can expedite arrival to the origin when $-1<\beta U_{0}<5$, but not when $\beta U_{0}>5$. The results presented herein generalize results for simple diffusion with resetting — a widely applicable model that is obtained from ours by setting $U_{0}=0$. Extending to general potential strengths, our work opens the door to theoretical and experimental investigation of a plethora of problems that bring together resetting and diffusion in logarithmic potential.
\end{abstract}

PACS numbers: 05.40.-a,05.40.Jc

\section{INTRODUCTION}

Resetting, i.e., stopping an ongoing dynamical process to start it anew, has recently gained significant attention due to its prevalence in natural and man made systems. For example, it has long been known that resetting certain computer algorithms can significantly enhance their performance ${ }^{1-4}$. Resetting is also commonly considered in the context of search processes 514 , e.g., foraging animals and birds come back to their dens and nests repeatedly $\sqrt{15 \mid 16}$; and inconvenient weather may force a team of rescuers to temporarily stop their search efforts and return to base ${ }^{10}$. Natural disasters and epidemics may also lead to resetting, e.g., by drastically reducing the population of a living species in a certain locality 17 ; and stock market crashes have a similar effect on asset prices 18 . At the microscopic level, resetting is an integral part of the well-known Michaelis-Menten reaction scheme of enzymatic catalysis $19-22$. This scheme is used to describe a variety of cellular processes ${ }^{23}$ ranging from RNA transcription 24 to facilitated diffusion ${ }^{25 \mid 26}$ and from the work of GTPase proteins 27 to chaperone assisted protein folding 28 . For all these reasons and others, resetting and its applications have now become a focal point of scientific interest 29 .

Diffusion with stochastic resetting serves as a paradigmatic model to understand resetting phenomena ${ }^{30}$. In this model, one considers a Brownian particle that returns to its initial position randomly in time. This resetting process drives the system away from equilibrium, giving rise to a nonequilibrium steady state and interesting relaxation dynamics. In the presence of an absorbing boundary, this model also provides a classical example of a system where resetting accelerates the

\footnotetext{
a)Electronic mail: somritaray@mail.tau.ac.i1

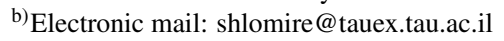

completion of a first-passage process $43[44$.

In many cases, diffusion occurs in the presence of a bias. A natural way to model such phenomena is to consider a Brownian particle in a suitable potential landscape. Similar to free-diffusion, resetting then leads to a nonequilibrium steady state 45 . The scenario gets more interesting in the presence of an absorbing boundary since resetting then plays a dual role: either facilitating or hindering the resulting firstpassage process 0 . Moreover, as system parameters are varied, resetting can invert its role thereby leading to a resetting transition 51920 . This transition, as well as the emergence of non-equilibrium steady states, were recently explored for diffusion in various potential landscapes, e.g., linear, harmonic and power-law 50 . An important potential landscape that was not considered despite its centrality is the logarithmic potential.

The logarithmic potential arises as an effective potential in a large variety of problems in Chemical, Statistical and Biological Physics. For example, in the denaturation process of double-stranded DNA, it appears as an entropic term in the free energy cost of unzipping DNA base-pairs that generate denaturation bubbles $51-56$. Diffusion in an effective logarithmic potential is a popular model to study the spreading of momenta of cold atoms trapped in optical lattices $57+64$. The interactions of colloids and polymers with walls of narrow channels and pores give rise to an "entropic" potential that is logarithmic 65 . 71 . Log potential also arises in Dyson's Brownian motion, where Coulomb gas is interpreted as a dynamical system to explore the eigenvalues of random matrices 7273 . Other well-known examples include systems with long-range interacting particles 74 , selfgravitating Brownian particles $5 / 76$, charges in the vicinity of a polyelectrolyte ${ }^{77 / 78}$ and interacting tracers in one-dimensional driven lattice gases 79 .

The logarithmic potential owns a central singularity at the origin, but behaves as a slowly varying function far away from 
it. Because of these unique features, diffusion in logarithmic potential leads to slow relaxation ${ }^{59|60| 80 \mid 81}$ and non-trivial first-passage properties 82 . A 4 . A Brownian particle in a logarithmic potential is therefore expected to serve as an interesting model to study the effects of resetting on stochastic dynamics.

In strongly repulsive logarithmic potential, a diffusing particle cannot reach the origin 82 . Stochastic resetting is then expected to lead to a non-equilibrium steady state. In stark contrast, when the logarithmic potential is either attractive or weakly repulsive, a diffusing particle will reach the origin in finite time ${ }^{82}$. Stochastic resetting can then either accelerate or delay the resulting first passage process. Going from the strongly repulsive case to the attractive case can be done by tuning a single model parameter - the strength of the potential in units of the thermal energy. Diffusion with resetting in a logarithmic potential thus lends itself as attractive model to study stochastic resetting and the range response to it. In what follows, we provide a detailed analysis of this model system.

The rest of this paper is organized as follows. We start in Sec. II where we revisit some earlier works and review the problem of diffusion in a logarithmic potential. In Sec. III, we explore the properties of the nonequilibrium steady state that the particle attains due to resetting while it diffuses in a strongly repulsive logarithmic potential. Considering the potential to be attractive/weakly repulsive, in Sec. IV we study the first-passage of the particle in the presence of resetting to an absorbing boundary placed at the origin. In the same section, we also explore the resetting transition. The final conclusions are drawn in Sec. V, where we construct a full phase diagram for the present problem.

\section{DIFFUSION IN A LOGARITHMIC POTENTIAL}

Diffusion in a logarithmic potential has attracted considerable attention in recent years $57+62 \mid 80-84$. Here, we present a brief review of the problem. Consider a particle undergoing diffusion in a potential $U(x)=U_{0} \log |x|$, where $U_{0}$ is a constant with dimensions of energy and $x$ is dimensionless. The potential is attractive for $U_{0}>0$ while it is repulsive for $U_{0}<0$. The diffusion constant $D$ is given by the EinsteinSmoluchowski relation $D=(\beta \zeta)^{-1}$, where $\beta$ is the thermodynamic beta and $\zeta$ stands for the friction coefficient. An absorbing boundary is placed at the origin such that the particle, starting from a position $x_{0}>0$, diffuses in the interval $0 \leq x<\infty$ until it hits the origin and is immediately removed from the system.

The time evolution of $p(x, t)$, the conditional probability density of finding the particle at position $x$ at time $t$ provided that the initial position was $x_{0}$, is then given by the FokkerPlanck equation

$$
\frac{\partial p(x, t)}{\partial t}=\frac{\partial}{\partial x}\left[\left(\frac{U_{0}}{\zeta x}\right) p(x, t)\right]+D \frac{\partial^{2} p(x, t)}{\partial x^{2}},
$$

where the initial condition is $p(x, 0)=\delta\left(x-x_{0}\right)$ and the boundary condition reads $p(0, t)=0$. Eq. (1) is exactly solvable ${ }^{82}$. To present the solution, first we define the parameter

$$
v:=\frac{1+\beta U_{0}}{2} .
$$

The solution to Eq. 11) then reads $82 \mid 84$,

$$
p(x, t)= \begin{cases}\frac{x}{2 D t}\left(\frac{x_{0}}{x}\right)^{v} \exp \left(-\frac{x^{2}+x_{0}^{2}}{4 D t}\right) I_{-v}\left(\frac{x x_{0}}{2 D t}\right) & \text { if } \beta U_{0}<-1, \\ \frac{x}{2 D t}\left(\frac{x_{0}}{x}\right)^{v} \exp \left(-\frac{x^{2}+x_{0}^{2}}{4 D t}\right) I_{v}\left(\frac{x x_{0}}{2 D t}\right) & \text { if } \beta U_{0} \geq-1,\end{cases}
$$

where $I_{ \pm v}(\cdot)$ is the modified Bessel function of the first kind with order $\pm v$.

For $\beta U_{0}<-1$, the potential is strongly repulsive. One then finds from Eq. (3) that $p(x, t)$ is always normalized, i.e., $\int_{0}^{\infty} p(x, t) d x=1$. Therefore, in this case, the particle never reaches the absorbing boundary at the origin. In stark contrast, for $\beta U_{0}>-1$, where the potential is either weakly repulsive or attractive, the particle eventually hits the absorbing boundary for every single realization. The survival probability $Q(t):=\int_{0}^{\infty} p(x, t) d x$, i.e., the probability that the particle is not absorbed at the origin by time $t$, is then given by 84

$$
Q(t)=1-\frac{\Gamma\left(v, \frac{x_{0}^{2}}{4 D t}\right)}{\Gamma(v)},
$$

where $\Gamma(v):=\int_{0}^{\infty} x^{\nu-1} e^{-x} d x$ and $\Gamma(v, a):=\int_{a}^{\infty} x^{\nu-1} e^{-x} d x$ denote the Gamma function and the upper incomplete Gamma function, respectively. The probability density function for the first-passage time, $T$, to the origin can be calculated from the survival probability as $f_{T}(t)=-\partial Q(t) / \partial t$, which gives $82 \mid 84$

$$
f_{T}(t)=\frac{t^{-(v+1)}}{\Gamma(v)}\left(\frac{x_{0}^{2}}{4 D}\right)^{v} \exp \left(-\frac{x_{0}^{2}}{4 D t}\right) .
$$

Note that in the long time limit $f_{T}(t) \propto t^{-(v+1)}$. Consequently in the same limit $Q(t) \propto t^{-v}$. Thus $v$ is known as the "persistence exponent" 44 , governing the decay of the survival probability. For free diffusion $U_{0}=0$, and Eq. (2) gives $v=1 / 2$. Eq. (5) then reduces to the well-known form $f_{T}(t)=\frac{x_{0}}{\sqrt{4 \pi D t^{3}}} \exp \left[-\frac{x_{0}^{2}}{4 D t}\right]$, which describes the first-passage time (FPT) distribution of a freely diffusing particle to the absorbing boundary 85 .

The fact that the system behaves differently depending on the potential makes the current problem a highly interesting one to study the effect of stochastic resetting. On one hand, when the particle diffuses in a strongly repulsive logarithmic potential $\left(\beta U_{0}<-1\right)$, its survival probability in the interval $[0, \infty]$ does not decay with time. Introduction of stochastic resetting is then expected to lead to a non-equilibrium steady state. On the other hand, when the particle diffuses in either attractive or weakly repulsive logarithmic potential $\left(\beta U_{0}>-1\right)$, its survival probability decays with time. Starting from $x_{0}>0$, the particle will now reach the origin in finite time. Introduction of resetting can then either expedite 


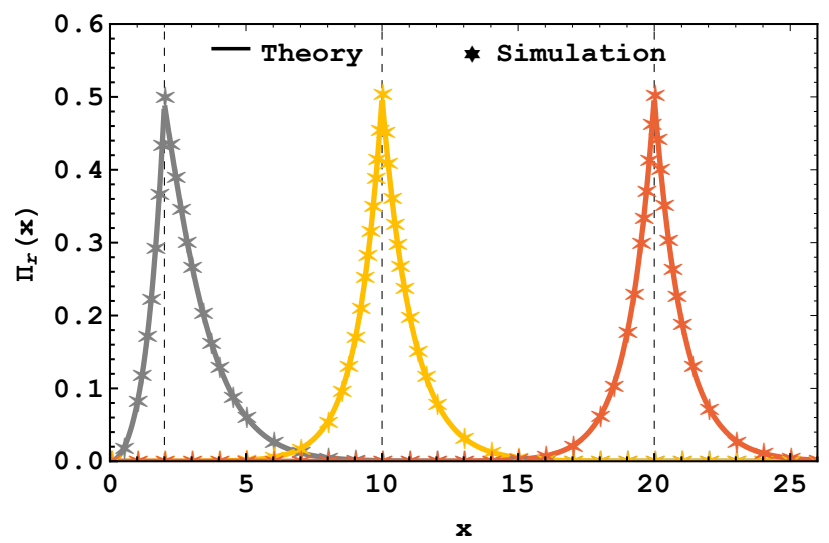

FIG. 1. The steady state distribution for diffusion in a strongly repulsive logarithmic potential under stochastic resetting. The theoretically predicted density $\Pi_{r}(x)$ from Eq. 10 is plotted for different values of the initial/resetting position $x_{0}=\{2,10,20\}$. The symbols showing simulation data are in excellent agreement with the theory. Here we have taken $\beta U_{0}=-2.0$ and $\alpha_{0}=1.0$.

or delay completion of this first-passage process ${ }^{10}$. Motivated by these different possible outcomes, we study the effects of stochastic resetting on diffusion in a logarithmic potential.

\section{DIFFUSION WITH RESETTING IN STRONGLY REPULSIVE LOGARITHMIC POTENTIAL}

In this Section, we explore the effect of stochastic resetting on a particle diffusing in a strongly repulsive logarithmic potential $\left(\beta U_{0}<-1\right)$. We assume that the particle is reset, i.e., taken back to its initial position $x_{0}$, with a constant rate $r$. This means that the times between two consecutive resetting events are taken from an exponential distribution with mean $1 / r$. Setting $p_{r}(x, t)$ as the conditional probability density of finding the particle at position $x$ at time $t$ provided that the initial position was $x_{0}$, the Fokker-Planck equation for the process with resetting reads

$$
\begin{array}{r}
\frac{\partial p_{r}(x, t)}{\partial t}=\frac{\partial}{\partial x}\left[\left(\frac{U_{0}}{\zeta x}\right) p_{r}(x, t)\right]+D \frac{\partial^{2} p_{r}(x, t)}{\partial x^{2}} \\
-r p_{r}(x, t)+r \delta\left(x-x_{0}\right),
\end{array}
$$

where $\delta\left(x-x_{0}\right)$ is a Dirac delta function. Note that in the absence of resetting $(r=0)$, Eq. (6) boils down to Eq. (1), the Fokker-Planck equation for the underlying process. For $r>0$, there is a loss of probability from position $x$ and a gain of probability at position $x_{0}$ due to resetting. The last two terms on the right hand side of Eq. (6) account for this additional probability flow, which is proportional to $r$, the resetting rate.

\section{A. Non-equilibrium steady state}

In what follows, we will be primarily interested in the steady state probability density of the process under stochastic resetting, $\Pi_{r}(x):=\lim _{t \rightarrow \infty} p_{r}(x, t)$. Since $d \Pi_{r}(x) / d t=0$,

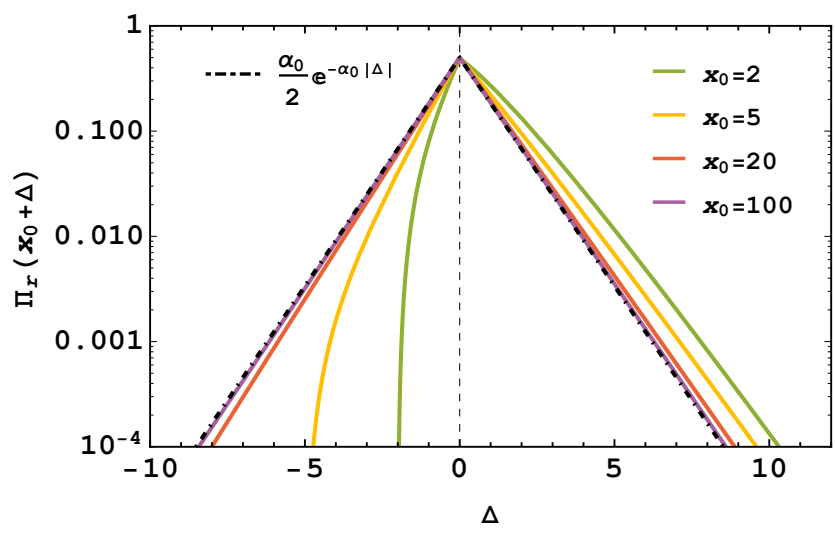

FIG. 2. The shifted steady state distribution $\Pi_{r}\left(x_{0}+\Delta\right)$ for different values of $x_{0}$, where $\Delta \in\left\{-x_{0}, \infty\right\}$. The solid lines are plotted using Eq. 10. The dot-dashed black line shows the asymptotic result of Eq. 11. Here we have taken $\beta U_{0}=-4.0$ and $\alpha_{0}=1.0$.

Eq. (6) gives

$$
\begin{array}{r}
\frac{d^{2} \Pi_{r}(x)}{d x^{2}}+\frac{d}{d x}\left[\left(\frac{\beta U_{0}}{x}\right) \Pi_{r}(x)\right] \\
-\left(\frac{r}{D}\right) \Pi_{r}(x)= \\
-\left(\frac{r}{D}\right) \delta\left(x-x_{0}\right) .
\end{array}
$$

The conventional way to calculate $\Pi_{r}(x)$ is to solve Eq. (7). We discuss this in detail in Appendix A. However, the solution can also be obtained directly by using a general relation that connects the propagator of an underlying stochastic process $p(x, t)$ with its steady state distribution under resetting, $\Pi_{r}(x)$. This relation is given by ${ }^{29}$

$$
\Pi_{r}(x)=\int_{0}^{\infty} r e^{-r t} p(x, t) d t=r \tilde{p}(x, r)
$$

where $\tilde{p}(x, s):=\int_{0}^{\infty} e^{-s t} p(x, t) d t$ denotes the Laplace transform of $p(x, t)$.

Therefore, in order to calculate $\Pi_{r}(x)$, we Laplace transform Eq. (3) for $\beta U_{0}<-1$ [Appendix B] and set $s=r$ to obtain

$\tilde{p}(x, r)= \begin{cases}\frac{x}{D}\left(\frac{x_{0}}{x}\right)^{v} I_{-v}\left(\sqrt{\frac{r x_{0}^{2}}{D}}\right) K_{-v}\left(\sqrt{\frac{r x^{2}}{D}}\right) \text { if } x \geq x_{0}, \\ \frac{x}{D}\left(\frac{x_{0}}{x}\right)^{v} K_{-v}\left(\sqrt{\frac{r x_{0}^{2}}{D}}\right) I_{-v}\left(\sqrt{\frac{r x^{2}}{D}}\right) \text { if } x<x_{0} .\end{cases}$

Plugging Eq. (9) into Eq. (8) and setting $\alpha_{0}:=\sqrt{r / D}$, we get the steady state density

$$
\Pi_{r}(x)= \begin{cases}\alpha_{0}^{2} x\left(\frac{x_{0}}{x}\right)^{v} I_{-v}\left(\alpha_{0} x_{0}\right) K_{-v}\left(\alpha_{0} x\right) & \text { if } x \geq x_{0}, \\ \alpha_{0}^{2} x\left(\frac{x_{0}}{x}\right)^{v} K_{-v}\left(\alpha_{0} x_{0}\right) I_{-v}\left(\alpha_{0} x\right) & \text { if } x<x_{0} .\end{cases}
$$

In Fig. 1. we plot $\Pi_{r}(x)$ for different values of the initial position $x_{0}$. The solid lines denote the analytical results, obtained by plotting Eq. 10 and the symbols are coming from 


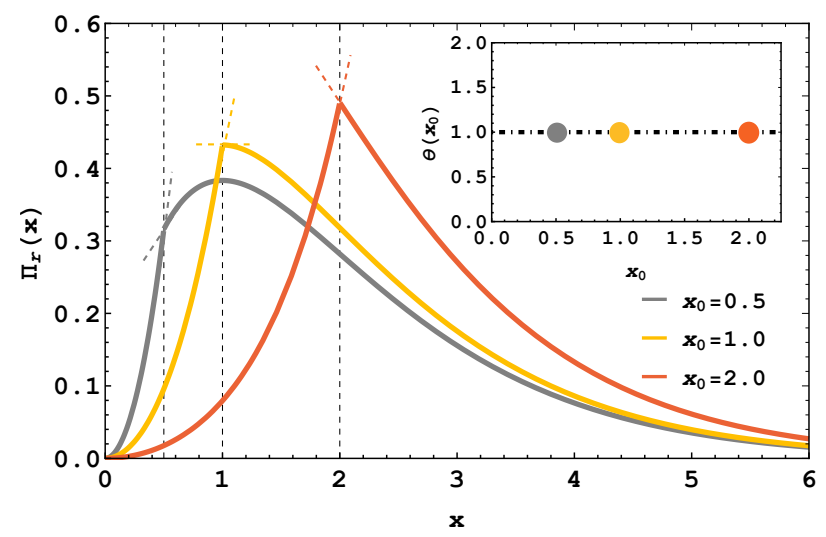

FIG. 3. Main: The steady state density $\Pi_{r}(x)$ for different values of the initial position $x_{0} . \Pi_{r}(x)$ has a cusp at $x_{0}$ that coincides with its mode for moderate to high values of $x_{0}$. For small values of $x_{0}$, the mode of the distribution appears to the right of the cusp. Inset: The discontinuity in $\partial \Pi_{r}(x) / \partial x$ at $x=x_{0}$, denoted $\theta\left(x_{0}\right)$ [Eq. [13], is a non-zero constant, $\alpha_{0}^{2}$. Here we have taken $\beta U_{0}=-2.0$ and $\alpha_{0}=1$.

Langevin dynamics simulations. The details of the numerical simulations are given in Appendix C.

Examining Fig. 1, we see that $\Pi_{r}(x)$ is asymmetric, which is particularly apparent for small values of $x_{0}$. The potential, being most repulsive at $x=0$, pushes the particle away from the origin thereby generating this asymmetry. The effect dies down for higher values of $x_{0}$, resulting in a steady state density that is almost symmetric. Indeed, when $x_{0} \gg \alpha_{0}^{-1}$, the particle is most likely to stay away from the origin, where the logarithmic potential varies slowly and is almost flat. This situation is similar to free diffusion. Therefore, in this limit $\Pi_{r}(x)$ is expected to closely resemble a Laplace distribution, which describes the steady state density for free diffusion with stochastic resetting $\frac{30}{\text {. }}$.

In order to explore this further, we set $x=x_{0}+\Delta$ such that $|\Delta| / x_{0} \ll 1$, and perform an asymptotic analysis of Eq. $10 p$ in the limit $x_{0} \gg \alpha_{0}^{-1}$. The limiting expressions of the modified Bessel functions for large arguments are $\lim _{y \rightarrow \infty} I_{-v}(y) \simeq$ $e^{y} / \sqrt{2 \pi y}$ and $\lim _{y \rightarrow \infty} K_{-v}(y) \simeq e^{-y} \sqrt{\pi / 2 y}$, respectively 8 . Utilizing these together with Eq. (10) we get

$$
\begin{aligned}
\Pi_{r}(x) & =\Pi_{r}\left(x_{0}+\Delta\right) \\
& \simeq \frac{\alpha_{0}}{2} e^{-\alpha_{0}|\Delta|}\left(\frac{x_{0}}{x_{0}+\Delta}\right)^{v-1} \frac{x_{0}}{\sqrt{x_{0}\left(x_{0}+\Delta\right)}}, \\
& \simeq \frac{\alpha_{0}}{2} e^{-\alpha_{0}\left|x-x_{0}\right|}
\end{aligned}
$$

where we have neglected all terms of order $|\Delta| / x_{0} \ll 1$. Eq. 11 proves that when $x_{0} \gg \alpha_{0}^{-1}$, the steady state distribution near $x_{0}$ converges to the Laplace distribution. In Fig. 2 , we plot the shifted steady state density $\Pi_{r}\left(x_{0}+\Delta\right)$ vs. $\Delta$ to explicitly show that it merges with that of free diffusion ${ }^{30}$ for sufficiently high values of $x_{0}$.

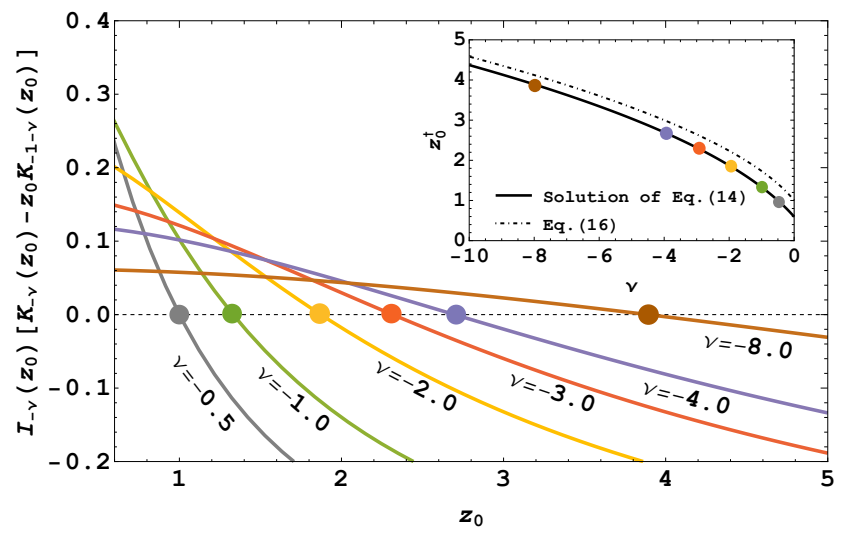

FIG. 4. Main: Graphical solution of Eq. 14 for different values of $v$. The solutions, $z_{0}^{\dagger}$, are highlighted by circles. Inset: Solid line shows the variation of $z_{0}^{\dagger}$ vs. $v$, with colored circles corresponding to the values of $v$ from the main figure. The dashed line shows the upper bound from Eq. 16.

\section{B. The cusp and the mode}

It is apparent from Fig. 1 and 2 that the steady state density has a cusp at the resetting position $x_{0}$. In what follows, we explore the cusp and the mode of the distribution in detail. In Fig. 3, we plot $\Pi_{r}(x)$ for some moderate values of $x_{0}$, highlighting the slopes from both sides of the cusp. Letting $\Pi_{r}^{-}(x)$ and $\Pi_{r}^{+}(x)$ denote the left and right branches of $\Pi_{r}(x)$, we obtain explicit expressions for these slopes

$$
\begin{aligned}
& \left.\frac{\partial \Pi_{r}^{+}(x)}{\partial x}\right|_{x \rightarrow x_{0}}=\alpha_{0}^{2} I_{-v}\left(\alpha_{0} x_{0}\right)\left[K_{-v}\left(\alpha_{0} x_{0}\right)-\alpha_{0} x_{0} K_{-1-v}\left(\alpha_{0} x_{0}\right)\right], \\
& \left.\frac{\partial \Pi_{r}^{-}(x)}{\partial x}\right|_{x \rightarrow x_{0}}=\alpha_{0}^{2} K_{-v}\left(\alpha_{0} x_{0}\right)\left[I_{-v}\left(\alpha_{0} x_{0}\right)+\alpha_{0} x_{0} I_{-1-v}\left(\alpha_{0} x_{0}\right)\right] .
\end{aligned}
$$

The difference between the slopes at $x_{0}$ in Eq. (12) then reads

$$
\theta\left(x_{0}\right)=\left[\frac{\partial \Pi_{r}^{-}(x)}{\partial x}-\frac{\partial \Pi_{r}^{+}(x)}{\partial x}\right]_{x \rightarrow x_{0}}=\alpha_{0}^{2},
$$

where we utilized the identity $K_{-v}(y) I_{-1-v}(y)+$ $I_{-v}(y) K_{-1-v}(y)=1 / y$. Thus, regardless of the particle's initial position, the difference between the slopes at $x_{0}$ is always $\alpha_{0}^{2}$, as is illustrated in the inset of Fig. 3 . This non-zero difference proves that $\Pi_{r}(x)$ always has a cusp at $x_{0}$. Note that Eq. (13) follows directly from Eq. (7), as we show in Appendix A.

It is evident from Fig. 3 that for moderate to high values of $x_{0}$, the mode of the distribution coincides with the cusp, whereas for small values of $x_{0}$, the mode appears to the right of the cusp. This effect arises due to the interplay between resetting and the action of the logarithmic potential. On one hand, resetting takes the particle back to $x_{0}$ and thus increases the probability density of finding it there. On the other hand, the strongly repulsive potential pushes the particle away from the origin, thereby decreasing the probability density of 


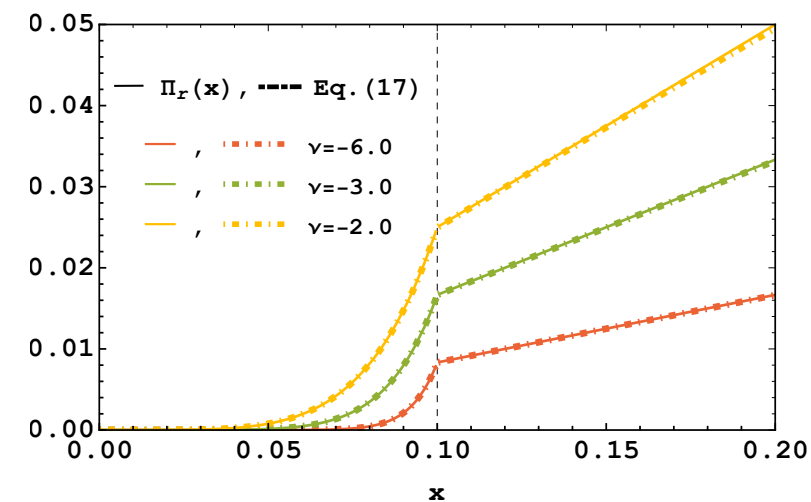

FIG. 5. The steady state distribution for different values of $v$ in the limit $x_{0} \ll \alpha_{0}^{-1}$. The solid lines denote $\Pi_{r}(x)$ from Eq. 10). The dash-dotted lines show limiting results from Eq. 17. Here we have taken $x_{0}=0.1$ and $\alpha_{0}=1.0$.

finding it close to $x=0$. For moderate to high values of $x_{0}$, the effect of resetting dominates: the cusp and the mode of $\Pi_{r}(x)$ coincide. In contrast, for small values of $x_{0}$ repulsion plays a significant role pushing the mode of the distribution to the right of the cusp.

In what follows, we will be interested in finding the minimal value of $x_{0}$, denoted $x_{0}^{\dagger}$, for which the cusp and the mode of $\Pi_{r}(x)$ coincide. To do that, we see from Fig. 3 that while the slope of $\Pi_{r}^{-}(x)$ is always positive at $x_{0}$, that of $\Pi_{r}^{+}(x)$ changes its sign. This transition happens at $x_{0}^{\dagger}$, where the slope of $\Pi_{r}^{+}(x)$ is exactly zero. Setting $\partial \Pi_{r}^{+}(x) / \partial x=0$ and utilizing Eq. (12) gives the following transcendental equation

$$
I_{-v}\left(z_{0}\right)\left[K_{-v}\left(z_{0}\right)-z_{0} K_{-1-v}\left(z_{0}\right)\right]=0,
$$

where $z_{0}:=\alpha_{0} x_{0}$. The solution of Eq. 14, denoted $z_{0}^{\dagger}$, can then be used to calculate the transition point

$$
x_{0}^{\dagger}=z_{0}^{\dagger} / \alpha_{0} .
$$

In Fig. 4, we graphically solve Eq. (14) for different values of $v$. In the inset, we plot the solutions, $z_{0}^{\dagger}$, vs $v \leq 0$. We find $z_{0}^{\dagger}$ to be a monotonically decreasing function of $v$. Thus recalling Eq. (2), we conclude that as the potential becomes more repulsive, the transition point $x_{0}^{\dagger}$ is pushed further away from the origin.

From Eq. (14), we see that at the transition point, $K_{-v}\left(z_{0}^{\dagger}\right) / K_{-1-v}\left(z_{0}^{\dagger}\right)=z_{0}^{\dagger}$, since $I_{-v}\left(z_{0}^{\dagger}\right) \neq 0$ for $z_{0}^{\dagger}>0$. The ratio of the modified Bessel functions of the second kind satisfies the inequality $K_{-v}(y) / K_{-1-v}(y)<(-v+$ $\left.\sqrt{v^{2}+y^{2}}\right) / y$, leading to an upper bound on $z_{0}^{\dagger}$

$$
z_{0}^{\dagger}<\sqrt{1-2 v}
$$

In the inset of Fig. 4, we plot this upper bound to show that Eq. (16) provides a simple yet effective way to locate the transition point $z_{0}^{\dagger}$, bypassing the solution of Eq. 14 .

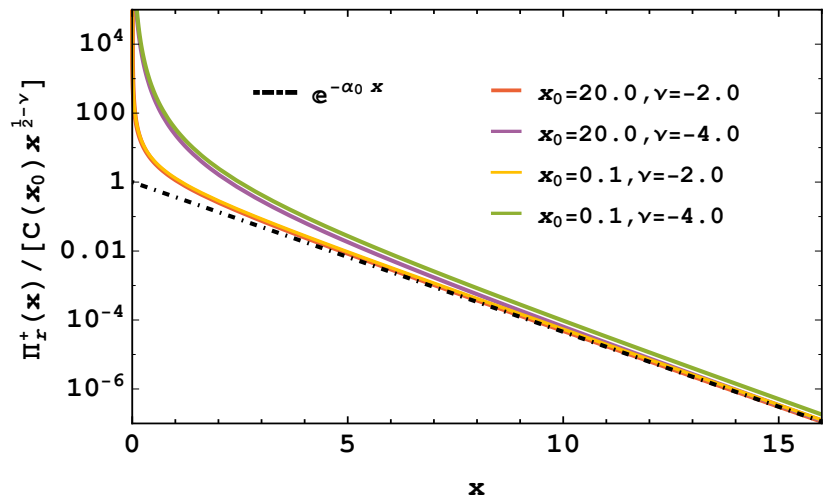

FIG. 6. The scaled steady state distribution $\Pi_{r}^{+}(x) /\left[C\left(x_{0}\right) x^{\frac{1}{2}-v}\right]$ for different values of $v$. Here, $\Pi_{r}^{+}(x)$ is taken from Eq. 10 and $C\left(x_{0}\right)$ is taken from Eq. 21. The dash-dotted black line shows the limiting expression $e^{-\alpha_{0} x}$ with $\alpha_{0}=1.0$.

\section{Asymptotic analysis}

Next, we analyze the behavior of the steady state density in the limit $x_{0} \ll \alpha_{0}^{-1}$, i.e., where $\Pi_{r}(x)$ is highly asymmetric. To explore this, we set $x=x_{0}+\Delta$ such that $|\Delta| \leq x_{0}$ and perform an asymptotic analysis of Eq. $\left[10\right.$ in the limit $x_{0} \ll \alpha_{0}^{-1}$. The limiting expressions of the modified Bessel functions for small arguments ${ }^{86} \operatorname{read} \lim _{y \rightarrow 0} I_{-v}(y) \simeq 2^{v} y^{-v} / \Gamma(1-v)$ and $\lim _{y \rightarrow 0} K_{-v}(y) \simeq 2^{-1-v} y^{v} \Gamma(-v)$, respectively. Utilizing these along with Eq. (10) we obtain

$$
\begin{aligned}
\Pi_{r}(x) & =\Pi_{r}\left(x_{0}+\Delta\right) \\
& \simeq\left\{\begin{array}{l}
-\frac{\alpha_{0}^{2}}{2 v} x \text { if } \Delta \geq 0, \\
x_{0} \ll \alpha_{0}^{-1} \\
-\frac{\alpha_{0}^{2} x_{0}^{2 v}}{2 v} x^{1-2 v} \text { if } \Delta<0 .
\end{array}\right.
\end{aligned}
$$

In Fig. 5, we plot the steady state distribution $\Pi_{r}(x)$ in the limit $x_{0} \ll \alpha_{0}^{-1}$ for different values of $v$. Agreement with Eq. (17) is clearly evident.

Finally, we explore how the steady state density decays at large values of $x$. We separate two cases; in the limit $x_{0} \gg \alpha_{0}^{-1}$, we recall that $\lim _{y \rightarrow \infty} I_{-v}(y) \simeq e^{y} / \sqrt{2 \pi y}$ and $\lim _{y \rightarrow \infty} K_{-v}(y) \simeq e^{-y} \sqrt{\pi / 2 y}$, and use Eq. 10 to obtain

$$
\Pi_{r}^{+}(x) \underset{\substack{x_{0} \gg \alpha_{0}^{-1} \\ x \rightarrow \infty}}{\simeq} \frac{\alpha_{0}}{2}\left(\frac{x}{x_{0}}\right)^{\frac{1}{2}-v} e^{-\alpha_{0}\left(x-x_{0}\right)} .
$$

On the other hand, in the limit $x_{0} \ll \alpha_{0}^{-1}$, utilizing the limiting expressions ${ }^{86} \lim _{y \rightarrow 0} I_{-v}(y) \simeq 2^{v} y^{-v} / \Gamma(1-v)$ and $\lim _{y \rightarrow \infty} K_{-v}(y) \simeq e^{-y} \sqrt{\pi / 2 y}$ together with Eq. 10 , we get

$$
\Pi_{r}^{+}(x) \underset{\substack{x_{0} \ll \alpha_{0}^{-1} \\ x \rightarrow \infty}}{\simeq} \frac{\sqrt{\pi} \alpha_{0}}{\Gamma(1-v)}\left[\frac{\alpha_{0} x}{2}\right]^{\frac{1}{2}-v} e^{-\alpha_{0} x} .
$$

Comparing Eq. (18) and (19), we see that in the large $x$ limit

$$
\Pi_{r}^{+}(x) \underset{x \rightarrow \infty}{\simeq} C\left(x_{0}\right) x^{\frac{1}{2}-v} e^{-\alpha_{0} x},
$$


where the prefactor is given by

$$
C\left(x_{0}\right)=\left\{\begin{array}{l}
\frac{\alpha_{0}}{2} x_{0}^{\nu-\frac{1}{2}} e^{\alpha_{0} x_{0}} \text { for } x_{0} \gg \alpha_{0}^{-1}, \\
\frac{\sqrt{\pi} \alpha_{0}}{\Gamma(1-v)}\left(\frac{\alpha_{0}}{2}\right)^{\frac{1}{2}-v} \text { for } x_{0} \ll \alpha_{0}^{-1} .
\end{array}\right.
$$

In Fig. 6, we plot the scaled steady state distribution $\Pi_{r}^{+}(x) /\left[C\left(x_{0}\right) x^{\frac{1}{2}-v}\right]$ to show that it always converges to the $v$-independent function $e^{-\alpha_{0} x}$ for large values of $x$.

\section{DIFFUSION WITH RESETTING IN WEAKLY REPULSIVE OR ATTRACTIVE LOGARITHMIC POTENTIAL}

In the previous section, we explored the non-equilibrium steady state attained for diffusion with resetting in a strongly repulsive logarithmic potential $\left(\beta U_{0}<-1\right)$. In contrast, when the potential is weakly repulsive or attractive $\left(\beta U_{0}>-1\right)$, a steady state is not achieved since the particle will eventually hit the absorbing boundary at the origir $\frac{82 \mid 84}{}$. Here we explore the effect of stochastic resetting on this first passage process.

\section{A. First-passage with resetting}

Consider a particle that diffuses in a potential $U(x)=$ $U_{0} \log |x|$ with $\beta U_{0}>-1$ and is subject to resetting with a constant rate $r$. The conventional way to calculate its firstpassage to the absorbing boundary at $x=0$ involves the backward Fokker Planck equation approach ${ }^{30}$. In this method, one treats the initial position $x_{0}$ as a variable. Hence, we assume that after each resetting event, the particle returns to $x_{r}>0$ (which in principle can be different than its initial position $x_{0}>0$ ), solve the problem with arbitrary $x_{0}$ and $x_{r}$ and eventually set $x_{r}=x_{0}$. Recalling that $p_{r}\left(x, t \mid x_{0}\right)$ is the probability density of finding the particle at position $x$ at time $t$, we see that its backward Fokker Planck equation ${ }^{29 \mid 90}$ reads

$$
\begin{aligned}
\frac{\partial p_{r}\left(x, t \mid x_{0}\right)}{\partial t} & =-\left(\frac{U_{0}}{\zeta x_{0}}\right) \frac{\partial p_{r}\left(x, t \mid x_{0}\right)}{\partial x_{0}}+D \frac{\partial^{2} p_{r}\left(x, t \mid x_{0}\right)}{\partial x_{0}^{2}} \\
& -r p_{r}\left(x, t \mid x_{0}\right)+r p_{r}\left(x, t \mid x_{r}\right) .
\end{aligned}
$$

The survival probability $Q_{r}\left(t \mid x_{0}\right):=\int_{0}^{\infty} p_{r}\left(x, t \mid x_{0}\right) d x$, i.e., the probability that the particle is not absorbed at $x=0$ by time $t$, then evolves in time following the master equation

$$
\begin{array}{r}
\frac{\partial Q_{r}\left(t \mid x_{0}\right)}{\partial t}=-\left(\frac{U_{0}}{\zeta x_{0}}\right) \frac{\partial Q_{r}\left(t \mid x_{0}\right)}{\partial x_{0}}+D \frac{\partial^{2} Q_{r}\left(t \mid x_{0}\right)}{\partial x_{0}^{2}} \\
-r Q_{r}\left(t \mid x_{0}\right)+r Q_{r}\left(t \mid x_{r}\right),
\end{array}
$$

where the initial condition is $Q_{r}\left(0 \mid x_{0}\right)=1$ and the boundary condition reads $Q_{r}(t \mid 0)=0$. Eq. 23 is exactly solvable and the solution allows us to calculate the distribution of the FPT for the process under resetting, bypassing the calculation of $p_{r}\left(x, t \mid x_{0}\right)$. This derivation is given in Appendix D.

An alternative way to calculate the FPT distribution is by utilizing the general theory of first-passage under resetting $7 / 10$.
This theory asserts that the FPT distribution of a process with a constant resetting rate $r$ can be expressed in terms of the FPT distribution of the process without resetting. Letting $T$ denote the FPT of a generic process and $T_{r}$ its FPT under resetting, we set $\tilde{T}(s):=\langle\exp (-s T)\rangle$ and $\tilde{T}_{r}(s):=\left\langle\exp \left(-s T_{r}\right)\right\rangle$ as the Laplace transforms of $T$ and $T_{r}$, respectively. One can then show that the following relation holds ${ }^{7}$

$$
\tilde{T}_{r}(s)=\frac{\tilde{T}(s+r)}{\frac{s}{s+r}+\frac{r}{s+r} \tilde{T}(s+r)} .
$$

Eq. 24) is completely general and we will now use it to analyze diffusion with resetting in weakly repulsive $\left(-1<\beta U_{0}<\right.$ $0)$ and attractive $\left(\beta U_{0}>0\right)$ logarithmic potential.

Consider first the problem without resetting. The distribution of the first passage time $T$ to the absorbing boundary at the origin is then given by Eq. (5). Laplace transforming Eq. (5) [Appendix E] we get

$$
\tilde{T}(s)=\frac{\left(\sqrt{s x_{0}^{2} / D}\right)^{v} K_{v}\left(\sqrt{s x_{0}^{2} / D}\right)}{2^{v-1} \Gamma(v)} .
$$

Here again, $K_{V}(\cdot)$ denotes the modified Bessel function of the second kind and $\Gamma(v)$ denotes the Gamma function. Plugging Eq. 25] into Eq. 24) we obtain

$$
\tilde{T}_{r}(s)=\frac{(s+r)\left(\alpha x_{0}\right)^{v} K_{v}\left(\alpha x_{0}\right)}{s 2^{v-1} \Gamma(v)+r\left(\alpha x_{0}\right)^{v} K_{v}\left(\alpha x_{0}\right)},
$$

where $\alpha=\sqrt{(s+r) / D}$. Finally, to get the survival, we recall that $Q_{r}\left(t \mid x_{0}\right)=\int_{t}^{\infty} f_{T_{r}}(t) d t$, with $f_{T_{r}}(t)$ standing for the probability density function of $T_{r}$. Thus, $\tilde{Q}_{r}\left(s \mid x_{0}\right)=\left[1-\tilde{T}_{r}(s)\right] / s$ which gives

$$
\tilde{Q}_{r}\left(s \mid x_{0}\right)=\frac{2^{v-1} \Gamma(v)-\left(\alpha x_{0}\right)^{v} K_{v}\left(\alpha x_{0}\right)}{s 2^{v-1} \Gamma(v)+r\left(\alpha x_{0}\right)^{v} K_{v}\left(\alpha x_{0}\right)} .
$$

Eq. (26) and Eq. 27p provide closed-form expressions for the FPT distribution and survival of our process in Laplace space.

\section{B. Mean and standard deviation}

In this subsection, we compute the mean and variance of the first passage time $T_{r}$. With these at hand, we characterize four distinct FPT phases for diffusion with resetting in weakly repulsive or attractive logarithmic potential. We start with the mean FPT, which for a generic process under stochastic resetting follows directly from Eq. 24$]^{7}$

$$
\left\langle T_{r}\right\rangle=-\left[d \tilde{T}_{r}(s) / d s\right]_{s=0}=\frac{1}{r}\left[\frac{1-\tilde{T}(r)}{\tilde{T}(r)}\right] .
$$

In our case, plugging Eq. 25] into Eq. 287, we obtain the mean FPT to the origin

$$
\left\langle T_{r}\right\rangle=\frac{1}{r}\left[\frac{2^{v-1} \Gamma(v)}{\left(\alpha_{0} x_{0}\right)^{v} K_{v}\left(\alpha_{0} x_{0}\right)}-1\right],
$$



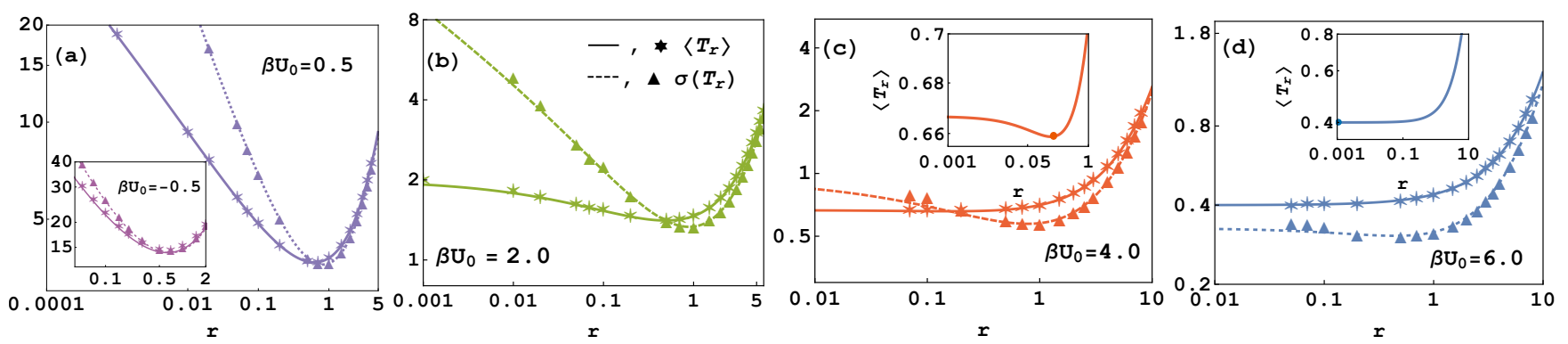

FIG. 7. The mean and the standard deviation of the FPT vs. the resetting rate, $r$, for four different phases of diffusion in a logarithmic potential. Lines indicate analytical results [solid lines: $\left\langle T_{r}\right\rangle$, dashed lines $\sigma\left(T_{r}\right)$ ] and symbols show data from simulations [stars: $\left\langle T_{r}\right\rangle$, triangles: $\sigma\left(T_{r}\right)$ ]. Panel (a): For $-1<\beta U_{0}<1$, the mean $\left\langle T_{r}\right\rangle$ and the standard deviation $\sigma\left(T_{r}\right)$ of the FPT show a non-monotonic variation with the resetting rate, and both diverge in the limit $r \rightarrow 0$ (no resetting). Panel (b): For $1<\beta U_{0}<3$, the mean and standard deviation of the FPT show a non monotonic variation with the resetting rate. However, while the mean FPT is finite in the limit $r \rightarrow 0$, the standard deviation diverges. Panel (c): For $3<\beta U_{0}<5$, the mean and the standard deviation of FPT still show a non monotonic variation with the resetting rate. Both are finite in the limit $r \rightarrow 0$, and $\sigma\left(T_{r=0}\right)>\left\langle T_{r=0}\right\rangle$. Panel (d): For $\beta U_{0}>5$, the mean FPT is monotonically increasing with the resetting rate. Both the mean and standard deviation are finite in the limit $r \rightarrow 0$, and $\sigma\left(T_{r=0}\right)<\left\langle T_{r=0}\right\rangle$. The insets of panels (c) and (d) present a zoom in on $\left\langle T_{r}\right\rangle$ from the corresponding main plots. In all panels we have taken $x_{0}=2.0$ and $D=1.0$.

with $\alpha_{0}=\sqrt{r / D}$, as introduced in Sec. III. Note that for $U_{0}=0$, i.e., free diffusion, $v=1 / 2$ [Eq. (2)]. The mean FPT then boils down to $\left\langle T_{r}\right\rangle=\left(e^{\sqrt{r x_{0}^{2} / D}}-1\right) / r$, which agrees with the result obtained by Evans and Majumdar for free diffusion with stochastic resetting 30 .

The second moment of the FPT can be calculated from Eq. [24 in a similar manner ${ }^{17}$

$$
\left\langle T_{r}^{2}\right\rangle=\left[d^{2} \tilde{T}_{r}(s) / d s^{2}\right]_{s=0}=2 \frac{r \frac{d \tilde{T}(r)}{d r}-\tilde{T}(r)+1}{r^{2}[\tilde{T}(r)]^{2}} .
$$

Plugging Eq. 25 into Eq. (30), one can calculate $\left\langle T_{r}^{2}\right\rangle$ and that in turn gives the standard deviation

$$
\begin{aligned}
& \sigma\left(T_{r}\right):=\sqrt{\left\langle T_{r}^{2}\right\rangle-\left\langle T_{r}\right\rangle^{2}} \\
= & \frac{1}{r} \sqrt{\frac{2^{v-1} \Gamma(v)\left[2^{v-1} \Gamma(v)-\left(\alpha_{0} x_{0}\right)^{1+v} K_{v-1}\left(\alpha_{0} x_{0}\right)\right]}{\left(\alpha_{0} x_{0}\right)^{2 v}\left[K_{v}\left(\alpha_{0} x_{0}\right)\right]^{2}}-1 .} .
\end{aligned}
$$

In Fig. 7 we plot the mean and the standard deviation of the FPT vs. the resetting rate $r$ for different values of $\beta U_{0} \in$ $\{-1, \infty\}$. We observe four distinct phases shown in panels (a), (b), (c) and (d). These show noticeably different behavior in the limit $r \rightarrow 0$. For weakly repulsive $(-1<\beta<0)$ or weakly attractive $\left(0<\beta U_{0}<1\right)$ potential, both the mean and standard deviation of the FPT diverge in the absence of resetting, i.e., for $r=0$ [panel (a)]. For weak to moderately attractive potential, where $1<\beta U_{0}<3$, the mean FPT in the absence of resetting is finite, but the standard deviation at $r=0$ diverges [panel (b)]. When the potential is moderately attractive $\left(3<\beta U_{0}<5\right)$, both $\left\langle T_{r}\right\rangle$ and $\sigma\left(T_{r}\right)$ become finite at $r=0$, with $\sigma\left(T_{r=0}\right)>\left\langle T_{r=0}\right\rangle$ [panel (c): main figure]. In this phase, the mean FPT still shows a non-monotonic behavior with the resetting rate [panel (c): inset]. Finally, for strongly attractive potential $\left(\beta U_{0}>5\right)$, we again find that both $\left\langle T_{r}\right\rangle$ and $\sigma\left(T_{r}\right)$ are finite in the absence of resetting, but in contrast to (c) here we have $\sigma\left(T_{r=0}\right)<\left\langle T_{r=0}\right\rangle$ [panel (d): main figure]. In this phase, $\left\langle T_{r}\right\rangle$ monotonically increases with $r$ [panel (d): inset].

To understand the behavior of the mean and standard deviation of the FPT in the limit $r \rightarrow 0$, we use Eq. (5) to calculate the moments of the FPT for the underlying process without resetting. The first moment $\langle T\rangle:=\int_{0}^{\infty} t f_{T}(t) d t$, i.e., the mean FPT, is found to diverge for weakly repulsive/attractive logarithmic potential, where $-1<\beta U_{0}<1$. Clearly, the standard deviation of the FPT, $\sigma(T):=\sqrt{\left\langle T^{2}\right\rangle-\langle T\rangle^{2}}$, also diverges in this regime, which supports our observation in panel (a). For $\beta U_{0}>1$, using Eq. (5) we obtain the following closed form expression for the mean FPT in the absence of resetting

$$
\langle T\rangle=\frac{x_{0}^{2}}{2 D\left(\beta U_{0}-1\right)},
$$

where we recall that $\beta U_{0}=2 v-1$ [see Eq. [2]]. Eq. [32] shows that $\langle T\rangle$ is finite for $\beta U_{0}>1$. The standard deviation, however, diverges whenever $\beta U_{0}<3$. Hence, for $1<\beta U_{0}<3$, the mean FPT is finite but $\sigma(T)$ diverges [panel (b)].

For $\beta U_{0}>3$, the standard deviation of the FPT for the process without resetting is finite and given by

$$
\sigma(T)=\frac{x_{0}^{2}}{\sqrt{2} D\left(\beta U_{0}-1\right) \sqrt{\beta U_{0}-3}} .
$$

In turn, the theory of first-passage with resetting asserts that the introduction of resetting will result in a decrease of the mean FPT whenever the ratio between the standard deviation and the mean of the FPT distribution - in the absence of resetting - is larger than unity, and vice versa ${ }^{7119}$. From Eqs. 32 and (33), we see that here this ratio, commonly known as the coefficient of variation $(\mathrm{CV})$, is given by

$$
C V(T)=\frac{\sigma(T)}{\langle T\rangle}=\sqrt{\frac{2}{\beta U_{0}-3}} .
$$




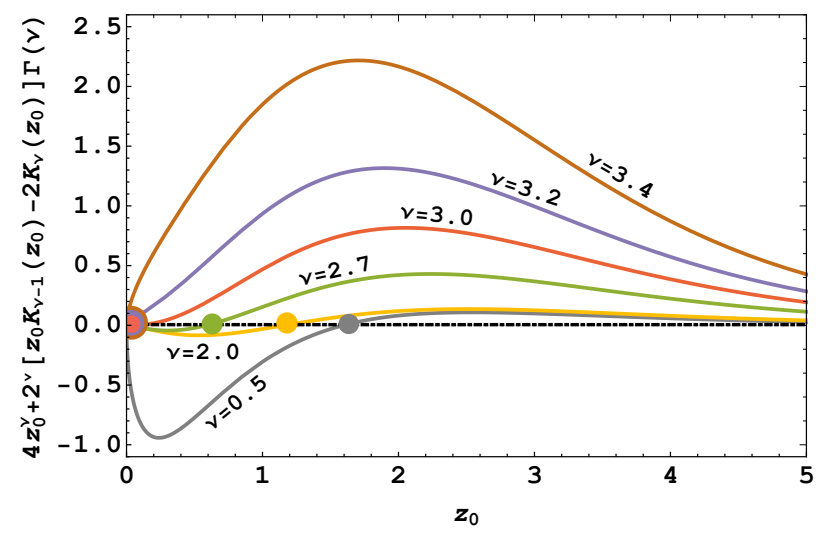

FIG. 8. Graphical solution of Eq. 37) for different values of $v$. The solutions, $z_{0}^{\star}$, are highlighted by circles.

From Eq. 34, it is evident that $C V(T)$ is greater than unity for $3<\beta U_{0}<5$, hence the introduction of resetting lowers the mean FPT in this case. In contrast, for $\beta U_{0}>5, C V(T)$ is less than unity [Eq. [34)]. Therefore, unlike previous cases, here the introduction of resetting hinders first-passage.

In addition to the analytical results of Eqs. (29) and (31), in Fig. 7 we also plot data for $\left\langle T_{r}\right\rangle$ and $\sigma\left(T_{r}\right)$ that are obtained by Langevin dynamics simulations. These data are in good agreement with theory. The details of the numerical simulation are given in Appendix C.

\section{The resetting transition}

In panels (a), (b) and (c), the variation of the mean FPT, $\left\langle T_{r}\right\rangle$, with the resetting rate $r$ is non-monotonic. The minimum of $\left\langle T_{r}\right\rangle$ in each of these cases is attained at an optimal resetting rate $r^{\star}>0$. In contrast, in panel (d), $\left\langle T_{r}\right\rangle$ shows a monotonic increase with $r$. Moreover, while $\left\langle T_{r}\right\rangle$ and $\sigma\left(T_{r}\right)$ intersect in panels (a), (b) and (c), they do not intersect in panel (d). It has recently been shown that for optimally restarted first-passage processes the mean is exactly equal to the standard deviation, i.e., $\left\langle T_{r^{\star}}\right\rangle=\sigma\left(T_{r^{\star}}\right)^{7}$. Therefore, the intersection points of $\left\langle T_{r}\right\rangle$ and $\sigma\left(T_{r}\right)$ in panel (a), (b) and (c) mark the optimal resetting rates. Summarizing, the results above prove that for weakly repulsive and weak to moderately attractive logarithmic potentials, the introduction of resetting accelerates first-passage to the origin, but when the attractive potential is sufficiently strong, resetting cannot expedite the process any more. This clearly indicates a resetting transition ${ }^{46}+48$ as $\beta U_{0}$ grows beyond a critical value. In this subsection, we discuss this transition in terms of the optimal resetting rate $r^{\star}$.

In order to explicitly calculate the optimal resetting rate, we recall from Sec. III that $z_{0}:=\alpha_{0} x_{0}=\sqrt{r x_{0}^{2} / D}$. Therefore,

$$
r=\frac{D z_{0}^{2}}{x_{0}^{2}}
$$

Plugging Eq. 235 into Eq. 29, we get the mean FPT in terms

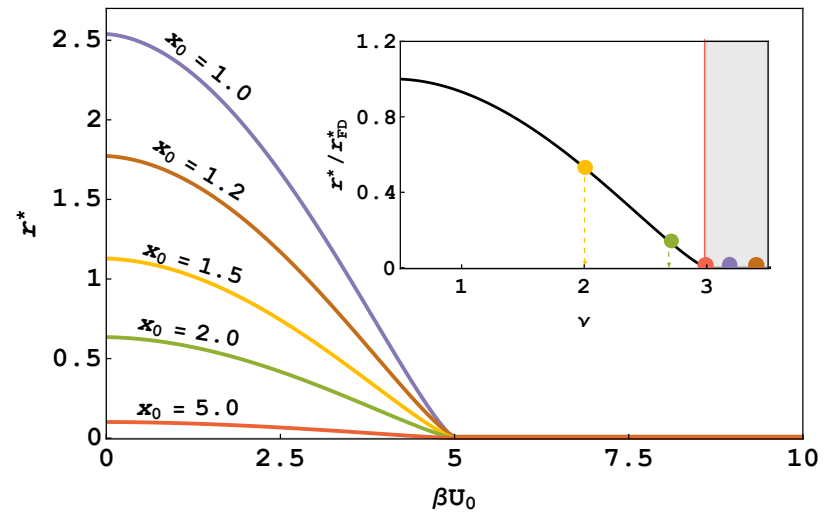

FIG. 9. Main: The optimal resetting rate $r^{\star}$ vs $\beta U_{0}$ for different values of $x_{0}$. The resetting transition is always observed at $\beta U_{0}=5$. Beyond this point, the optimal resetting rate is zero, i.e., resetting cannot expedite first passage of the particle to the origin. Inset: The scaled optimal resetting rate $r^{\star} / r_{F D}^{\star}$ vs. the persistence exponent $v=$ $\left(1+\beta U_{0}\right) / 2$. The resetting transition is observed at $v=3$, shown by the vertical orange-red line. The colored circles indicate different values of $v$ from Fig. 8

of $z_{0}$ as

$$
\left\langle T_{r}\right\rangle=\frac{x_{0}^{2}}{D z_{0}^{2}}\left[\frac{2^{v-1} \Gamma(v)}{z_{0}^{v} K_{v}\left(z_{0}\right)}-1\right] .
$$

When the process is reset at an optimal rate, $r=r^{\star}$, the mean FPT is minimized hence $\left[d\left\langle T_{r}\right\rangle / d r\right]_{r=r^{\star}}=0$. From Eq. (35) we get $d\left\langle T_{r}\right\rangle / d r \equiv\left(x_{0}^{2} / 2 z_{0} D\right) d\left\langle T_{r}\right\rangle / d z_{0}$, which at the optimal resetting rate leads to the following transcendental equation

$$
4 z_{0}^{v}+2^{v}\left[z_{0} K_{v-1}\left(z_{0}\right)-2 K_{v}\left(z_{0}\right)\right] \Gamma(v)=0 .
$$

In Fig. 8, we graphically solve Eq. 37 for different values of $v$. The solutions, denoted $z_{0}^{\star}$, can then be utilized to calculate the optimal resetting rates $r^{\star}$ following Eq. 35. In Fig. 9 we plot these optimal resetting rates vs. $\beta U_{0}$ for different values of the initial position $x_{0}$. It is evident from the plot that the point of the resetting transition is always at $\beta U_{0}=5$, which does not depend on $x_{0}$. In other words, the introduction of resetting expedites first passage only when the ratio between the strength of the potential and the thermal energy $\left(\beta^{-1}\right)$ is less than a certain critical value, $\beta U_{0}<5$. This critical value is universal in the sense that it is not affected by the initial distance of the particle from the absorbing boundary. This supports the discussion following Eq. (34) which marks the transition at $C V(T)=1$. In the inset of Fig. 9, we plot the optimal resetting rate scaled by $r_{F D}^{\star}$, the optimal resetting rate for free diffusion $\left(U_{0}=0\right)$. It can be seen that after this scaling, the optimal resetting rate is uniquely determined by the persistence exponent $v=\left(1+\beta U_{0}\right) / 2$.

\section{CONCLUSIONS}

In this work, we presented a comprehensive analysis of the effect of stochastic resetting on diffusion in a logarithmic 


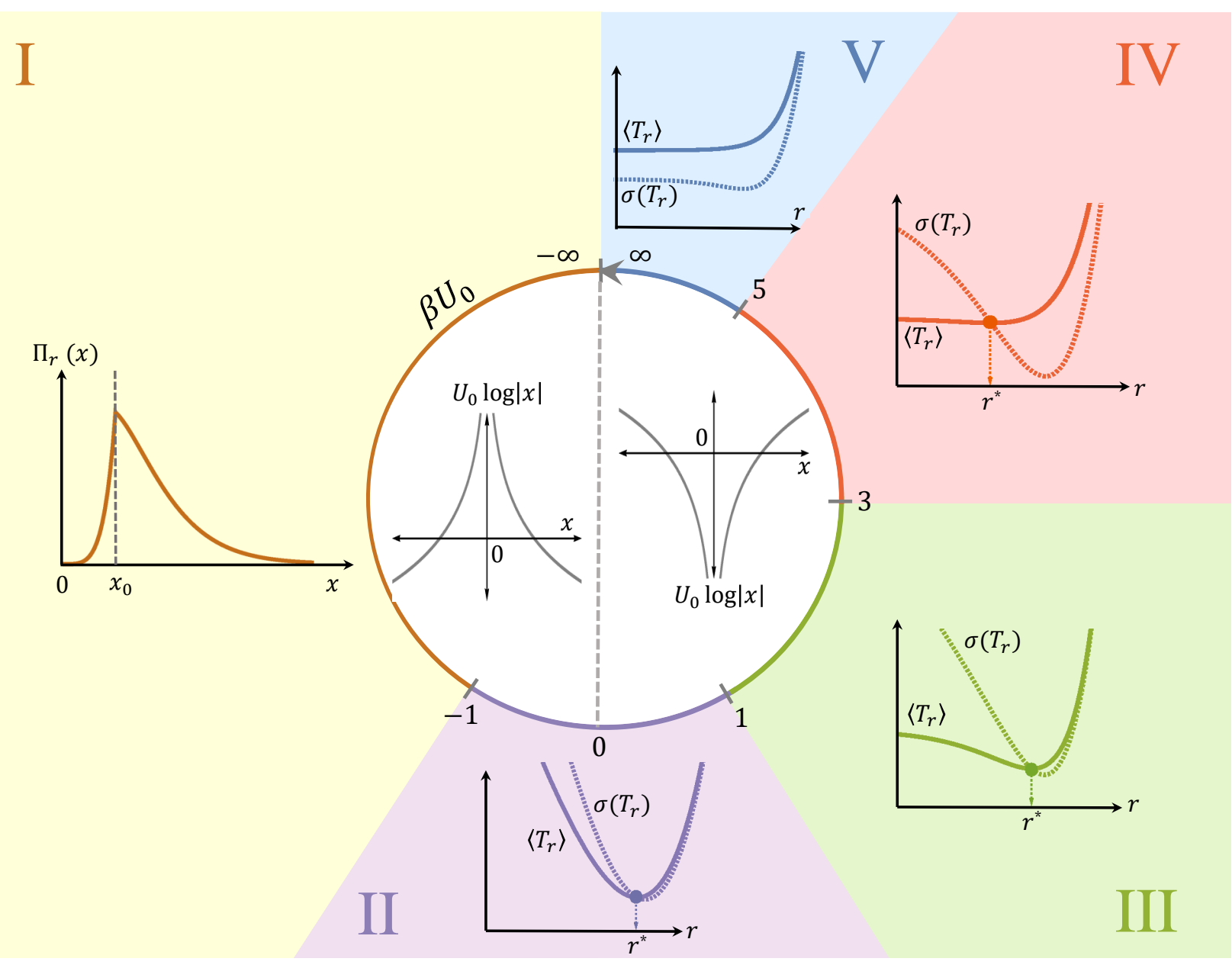

FIG. 10. A phase diagram illustrating the possible effects of stochastic resetting on diffusion in a logarithmic potential $U(x)=U_{0} \log |x|$. The potential is repulsive for $U_{0}<0$ and attractive for $U_{0}>0$, as shown in the inner circle. Phase transitions occur as $\beta U_{0}$, the ratio between the potential strength and the thermal energy, is tuned. Phase I: A particle, starting from $x_{0}$, will never reach the origin. Resetting the particle to $x_{0}$ at a rate $r$ then results in a nonequilibrium steady state. Phases II-V: A particle, starting from $x_{0}$, will eventually reach the origin. However, in the different phases the behaviour of the mean, $\left\langle T_{r}\right\rangle$, and standard deviation, $\sigma\left(T_{r}\right)$, of the first-passage time is markedly different in the limit $r \rightarrow 0$ as elaborately discussed in the main text. In particular, while in phases II-IV the introduction of resetting lowers the mean first passage time, i.e., expedites first passage to the origin, in phase $\mathrm{V}$ it is the other way around. The transition between these two markedly different behaviours happens at $\beta U_{0}=5$ irrespective of the particle's initial position.

potential $U(x)=U_{0} \log |x|$. Here $U_{0}$ denotes the strength of the potential which is attractive for $U_{0}>0$ and repulsive for $U_{0}<0$. We found that the effect of resetting on the dynamics of a particle that diffuses in this potential is guided solely by the interplay between this attraction/repulsion energy, $U_{0}$, and the thermal energy $\beta^{-1}$. This allows us to construct a phase diagram where transitions between phases occur as the dimensionless parameter $\beta U_{0}$ is tuned.

In Fig. 10, we show that the entire range $\beta U_{0} \in\{-\infty, \infty\}$ can be divided into five distinct phases. For $-\infty<\beta U_{0}<-1$, i.e., for a strongly repulsive potential, the diffusing particle can never reach the origin. In other words, the total probability of finding the particle in the ray $(0, \infty)$ [assuming it started its motion at $x_{0}>0$ ] does not decay with time. The introduction of resetting in this case leads to a steady state which marks phase I in Fig. 10 Detailed analysis of this steady state was provided in Section III.
For $\beta U_{0}>-1$, i.e., when the potential is either weakly repulsive or attractive, the particle is assured to eventually hit the absorbing boundary at the origin. Four phases can then be told apart. For $-1<\beta U_{0}<1$ (weakly repulsive/attractive potential) both the mean and the standard deviation of the FPT diverge in absence of resetting, which marks phase II. For $1<\beta U_{0}<3,\left\langle T_{r=0}\right\rangle$ is finite, but the standard deviation diverges as $r \rightarrow 0$, marking phase III. For $\beta U_{0}>3$, both the mean and the standard deviation of the FPT in absence of resetting are finite. However, while for $3<\beta U_{0}<5$, the standard deviation is greater than the mean (phase IV), for $5<\beta U_{0}<\infty$ it is the other way around (phase $\mathrm{V}$ ).

Following the general theory of first-passage with resetting ${ }^{7}$, we can summarize the observations as follows. For phases II, III and IV, i.e., for weakly repulsive or weak to moderately attractive potentials, introduction of resetting accelerates the first-passage of the particle to the origin. In these 
phases, the $\left\langle T_{r}\right\rangle$ and $\sigma\left(T_{r}\right)$ curves intersect each other at an optimal resetting rate $r^{\star}$, which minimizes the MFPT. In marked contrast, for phase $\mathrm{V}$, i.e., for a strongly attractive potential, resetting only delays first-passage to the origin. In this phase, the $\left\langle T_{r}\right\rangle$ and $\sigma\left(T_{r}\right)$ curves do not intersect.

The effect of resetting on the first-passage of a physical system can, in principle, be inverted by varying the system parameters, e.g. temperature, concentration etc., across some critical point, which gives rise to a "resetting transition" 46 . 48 . For diffusion in a potential $U(x)=U_{0} \log |x|$, we showed that the first-passage to the origin can be accelerated by resetting only when $\beta U_{0}<5$. This marks the point of the resetting transition at $\beta U_{0}=5$, which is the boundary between phase IV and phase V. For the current problem, this transition point is universal in the sense that it is independent of the initial distance of the particle from the absorbing boundary.

\section{ACKNOWLEDGEMENTS}

S. Ray acknowledges support from the Raymond and Beverly Sackler Center for Computational Molecular and Materials Science at Tel Aviv University. S. Reuveni acknowledges support from the Azrieli Foundation, from the Raymond and Beverly Sackler Center for Computational Molecular and Materials Science at Tel Aviv University, and from the Israel Science Foundation (grant No. 394/19). S. Ray is thankful to Arnab Pal for insightful discussions. The authors thank Arnab Pal and Sarah Kostinski for reading and commenting on early versions of this manuscript.

\section{APPENDIX A: STEADY STATE DENSITY BY SOLVING EQ. (7)}

Eq. (7) in the main text is a second-order, linear and inhomogeneous differential equation. Here, we solve Eq. (7) for $\Pi_{r}(x)$, the steady state density for diffusion under resetting in a strongly repulsive logarithmic potential $\left(\beta U_{0}<-1\right.$, i.e., $v<0$ [see Eq. [2]]). To do that, we perform the following variable transformation

$$
\Pi_{r}(x) \equiv x^{\rho} y(x)
$$

Plugging Eq. A.38 into Eq. 77 and setting $\alpha_{0}=\sqrt{r / D}$ we obtain

$$
\frac{d^{2} y(x)}{d x^{2}}+c_{1}\left(\frac{1}{x}\right) \frac{d y(x)}{d x}+\left[\frac{c_{2}}{x^{2}}-\alpha_{0}^{2}\right] y(x)=-\alpha_{0}^{2} \frac{\delta\left(x-x_{0}\right)}{x^{\rho}}
$$

where $c_{1}=\left(2 \rho+\beta U_{0}\right)$, and $c 2=(\rho-1)\left(\rho+\beta U_{0}\right)$. In Eqs. A.38 and A.39, $\rho$ is an arbitrary parameter. Setting $c_{1}=1$, we get $\rho=\left(1-\beta U_{0}\right) / 2 \equiv(1-v)$, which gives $c_{2}=-[(1+$ $\left.\left.\beta U_{0}\right) / 2\right]^{2} \equiv-v^{2}$. Therefore, Eq. A.39 reduces to

$$
\frac{d^{2} y(x)}{d x^{2}}+\left(\frac{1}{x}\right) \frac{d y(x)}{d x}-\left[\frac{v^{2}}{x^{2}}+\alpha_{0}^{2}\right] y(x)=-\alpha_{0}^{2} x^{v-1} \delta\left(x-x_{0}\right) .
$$

Eq. A.40 is an inhomogeneous modified Bessel equation; its general solution $\frac{87}{}$ is given by

$$
y(x)=\left\{\begin{array}{l}
A_{1} I_{-v}\left(\alpha_{0} x\right)+B_{1} K_{-v}\left(\alpha_{0} x\right) \text { for } x \geq x_{0}, \\
A_{2} I_{-v}\left(\alpha_{0} x\right)+B_{2} K_{-v}\left(\alpha_{0} x\right) \text { for } x<x_{0},
\end{array}\right.
$$

where $I_{-v}(\cdot)$ and $K_{-v}(\cdot)$ are modified Bessel functions of the first and second kind with order $v<0$. Since $\Pi_{r}(x)=$ $x^{1-v} y(x)$, the general solution of Eq. 77 reads

$$
\begin{aligned}
& \Pi_{r}^{+}(x)=A_{1} x^{1-v} I_{-v}\left(\alpha_{0} x\right)+B_{1} x^{1-v} K_{-v}\left(\alpha_{0} x\right) \text { for } x \geq x_{0}, \\
& \Pi_{r}^{-}(x)=A_{2} x^{1-v} I_{-v}\left(\alpha_{0} x\right)+B_{2} x^{1-v} K_{-v}\left(\alpha_{0} x\right) \text { for } x<x_{0} .
\end{aligned}
$$

where $\Pi_{r}^{+}(x)$ and $\Pi_{r}^{-}(x)$ denote the left and right branches of $\Pi_{r}(x)$, respectively. In order to find out the specific solution of Eq. A.42, we need to calculate the coefficients $A_{1}, B_{1}, A_{2}$ and $B_{2}$ explicitly, which we accomplish in the following way.

The steady state density $\Pi_{r}(x)$ must not diverge at $x \rightarrow \infty$, and that sets $A_{1}=0$.

In addition, $\Pi_{r}(x)$ must be continuous at $x=x_{0}$, i.e., $\Pi_{r}^{+}\left(x_{0}\right)=\Pi_{r}^{-}\left(x_{0}\right)$. This leads to

$$
B_{1}=B_{2}+A_{2}\left[\frac{I_{-v}\left(\alpha_{0} x_{0}\right)}{K_{-v}\left(\alpha_{0} x_{0}\right)}\right] .
$$

The third condition comes from integrating Eq. (7) over the narrow spatial interval $\left[x_{0}-\Delta, x_{0}+\Delta\right]$, where $|\Delta| / x_{0} \ll 1$, which gives

$$
\begin{aligned}
{\left[\frac{d \Pi_{r}(x)}{d x}\right]_{x_{0}-\Delta}^{x_{0}+\Delta}+\beta U_{0}\left[\frac{\Pi_{r}(x)}{x}\right]_{x_{0}-\Delta}^{x_{0}+\Delta} } & -\alpha_{0}^{2} \int_{x_{0}-\Delta}^{x_{0}+\Delta} \Pi_{r}(x) d x \\
& =-\alpha_{0}^{2}
\end{aligned}
$$

Here we utilize the identity $\int_{x_{0}-\Delta}^{x_{0}+\Delta} \delta\left(x-x_{0}\right)=1$. The condition for continuity at $x=x_{0}$ leads to $\lim _{\Delta \rightarrow 0}\left[\Pi_{r}(x) / x\right]_{x_{0}-\Delta}^{x_{0}+\Delta}=0$ and $\lim _{\Delta \rightarrow 0} \int_{x_{0}-\Delta}^{x_{0}+\Delta} \Pi_{r}(x) d x=0$. Therefore, Eq. A.44 boils down to

$$
\lim _{\Delta \rightarrow 0}\left[\frac{d \Pi_{r}(x)}{d x}\right]_{x_{0}-\Delta}^{x_{0}+\Delta} \equiv\left[\frac{d \Pi_{r}^{+}(x)}{d x}-\frac{d \Pi_{r}^{-}(x)}{d x}\right]_{x \rightarrow x_{0}}=-\alpha_{0}^{2} .
$$

Note that Eq. A.45) is the same as Eq. (13) in the main text, derived in a separate context. Calculating the slopes of $\Pi_{r}^{ \pm}(x)$ at $x=x_{0}$ from Eq. A.42 and plugging the results in Eq. A.45) we get

$$
\begin{aligned}
\left(B_{2}-B_{1}\right)\left[K_{-v}\left(\alpha_{0} x_{0}\right)\right. & \left.-\alpha_{0} x_{0} K_{-v-1}\left(\alpha_{0} x_{0}\right)\right]+A_{2}\left[I_{-v}\left(\alpha_{0} x_{0}\right)\right. \\
& \left.+\alpha_{0} x_{0} I_{-v-1}\left(\alpha_{0} x_{0}\right)\right]=\alpha_{0}^{2} x_{0}^{v} .
\end{aligned}
$$

The fourth and final condition comes from the fact that for $v<0$, the diffusing particle never reaches the absorbing boundary placed at the origin. Hence, $\Pi_{r}(x)$ is normalized to unity, i.e., $\int_{0}^{\infty} \Pi_{r}(x) d x \equiv \int_{0}^{x_{0}} \Pi_{r}^{-}(x) d x+\int_{x_{0}}^{\infty} \Pi_{r}(x) d x=1$, which leads to

$$
\begin{aligned}
B_{2} \int_{0}^{x_{0}} x^{1-v} K_{-v}\left(\alpha_{0} x\right)+\frac{x_{0}^{1-v}}{\alpha_{0}}\left[A_{2} I_{1-v}\left(\alpha_{0} x_{0}\right)\right. & \left.+B_{1} K_{1-v}\left(\alpha_{0} x_{0}\right)\right] \\
& =1,
\end{aligned}
$$


since $\quad \int_{0}^{x_{0}} x^{1-v} I_{-v}\left(\alpha_{0} x\right) d x=\alpha_{0}^{-1} x_{0}^{1-v} I_{1-v}\left(\alpha_{0} x_{0}\right)$ and $\int_{x_{0}}^{\infty} x^{1-v} K_{-v}\left(\alpha_{0} x\right) d x=\alpha_{0}^{-1} x_{0}^{1-v} K_{1-v}\left(\alpha_{0} x_{0}\right)$. Utilizing the general relation $I_{\mu}(y) K_{\mu+1}(y)+K_{\mu}(y) I_{\mu+1}(y)=1 / y$ that is valid for $\mu \in \mathbb{C}$, we solve Eqs. A.43, A.46, and A.47) to get the explicit expressions for $A_{2}, B_{1}$ and $B_{2}$

$$
\begin{aligned}
& B_{1}=\alpha_{0}^{2} x_{0}^{v} I_{-v}\left(\alpha_{0} x_{0}\right) \\
& A_{2}=\alpha_{0}^{2} x_{0}^{v} K_{-v}\left(\alpha_{0} x_{0}\right) \\
& B_{2}=0 .
\end{aligned}
$$

Plugging Eq. A.48 into Eq. A.42, we get Eq. 10] in the main text.

\section{APPENDIX B: LAPLACE TRANSFORM OF EQ. (3) FOR STRONGLY REPULSIVE POTENTIAL}

Eq. (3) in the main text gives $p(x, t)$, the probability density of finding a particle at position $x$ at time $t$, when the particle diffuses in a logarithmic potential. Here, we calculate the Laplace transform of Eq. (3) for $\beta U_{0}<-1(v<0)$, i.e., when the potential is strongly repulsive. Letting $\tilde{p}(x, s):=$ $\int_{0}^{\infty} e^{-s t} p(x, t) d t$ denote the Laplace transform of $p(x, t)$, we see that for $v<0$

$\tilde{p}(x, s)=\frac{x}{2 D}\left(\frac{x_{0}}{x}\right)^{v} \int_{0}^{\infty} \frac{e^{-s t}}{t} \exp \left(-\frac{x^{2}+x_{0}^{2}}{4 D t}\right) I_{-v}\left(\frac{x x_{0}}{2 D t}\right) d t$

Next, we note the identity

$$
\begin{aligned}
& \int_{0}^{\infty} e^{-s t}\left(\frac{1}{t}\right) \exp \left(-\frac{a+b}{2 t}\right) I_{\mu}\left(\frac{a-b}{2 t}\right) d t \\
& =2 K_{\mu}(\sqrt{s}(\sqrt{a}+\sqrt{b})) I_{\mu}(\sqrt{s}(\sqrt{a}-\sqrt{b}))
\end{aligned}
$$

which holds for $\operatorname{Re}(a) \geq \operatorname{Re}(b)>0$. Setting $\mu=-v$ in Eq. (B.50), we compare the integral with that in Eq. (B.49) to identify $(a+b) \equiv\left(x^{2}+x_{0}^{2}\right) / 2 D$ and $(a-b) \equiv\left(x x_{0}\right) / D$, which gives

$$
\sqrt{a}=\frac{\left(x+x_{0}\right)}{2 \sqrt{D}} \text { and } \sqrt{b}= \pm \frac{\left(x-x_{0}\right)}{2 \sqrt{D}} .
$$

Therefore, when $x \geq x_{0}, \sqrt{b}=\left(x-x_{0}\right) / 2 \sqrt{D}$ and when $x<x_{0}$, $\sqrt{b}=\left(x_{0}-x\right) / 2 \sqrt{D}$, leading to

$$
\begin{aligned}
& (\sqrt{a}+\sqrt{b})=\frac{x}{\sqrt{D}}, \quad(\sqrt{a}-\sqrt{b})=\frac{x_{0}}{\sqrt{D}} \text { for } x \geq x_{0} \\
& (\sqrt{a}+\sqrt{b})=\frac{x_{0}}{\sqrt{D}}, \quad(\sqrt{a}-\sqrt{b})=\frac{x}{\sqrt{D}} \text { for } x<x_{0}
\end{aligned}
$$

Plugging these into Eq. B.50 and then substituting the integral in Eq. B.49) accordingly, we get Eq. (9) in the main text by setting $s=r$.

\section{APPENDIX C: DETAILS OF NUMERIC SIMULATIONS}

The Langevin description for a particle diffusing in a potential $U(x)=U_{0} \log |x|$ in the overdamped limit reads

$$
\dot{x}=-\frac{U_{0}}{\zeta x}+\eta(t)
$$

where $\zeta$ is the friction coefficient and $\eta(t)$ denotes a white Gaussian noise with zero mean, i.e., $\langle\eta(t)\rangle=0$ and $\left\langle\eta(t) \eta\left(t^{\prime}\right)\right\rangle=2 D \delta\left(t-t^{\prime}\right)$. Here we numerically solve the above stochastic differential equation under resetting with a constant rate $r$. This implies that the position of the particle $x$ is reset to $x_{0}$ following random time intervals whose lengths are taken from an exponential distribution with mean $r^{-1}$.

To numerically calculate the steady state density $\Pi_{r}(x)$, we utilize Heun's method (commonly known as the second order Runge-Kutta method $)^{91}$ with step size $h=10^{-3}$ to perform the Langevin dynamics simulation and obtain the position $x$ of the particle in the long time limit for $10^{6}$ realizations. The steady state distribution is then estimated from the data and compared against the analytical results of Eq. [10] in Fig. 1 . The theoretical results and the simulation data are in excellent agreement.

To numerically calculate the mean, $\left\langle T_{r}\right\rangle$, and the standard deviation, $\sigma\left(T_{r}\right)$, of the FPT to the origin, we perform the Langevin dynamics simulation utilizing Heun's method with step size $h=10^{-4}$ or $h=10^{-6}$, depending on the system parameters. Smaller step size is necessary to minimize errors in the following cases. For weakly repulsive potential, i.e., $-1<\beta U_{0}<0$, smaller step size is required to lower down the otherwise high probability of losing the particle downhill when it hits the absorbing boundary at the origin but not immediately detected. For strongly attractive potential, $\beta U_{0}>5$, the first passage time $T_{r}$ reduces considerably in magnitude, which might lead to large errors if the step size is not reduced accordingly. The simulation is performed for $10^{6}$ realizations in each case to calculate the mean and the standard deviation of the FPT. The simulation data show very good agreement with the analytical results of Eq. (29) and Eq. (31), as shown in Fig. 7 .

\section{APPENDIX D: FIRST PASSAGE TIME BY SOLVING} EQ. (23)

Eq. (23) in the main text is a second order, linear and inhomogeneous partial differential equation. It describes the time evolution of $Q_{r}\left(t \mid x_{0}\right)$, the survival probability of a particle that diffuses under resetting in a weakly repulsive or attractive logarithmic potential $\left(\beta U_{0}>-1\right)$, in the presence of an absorbing boundary placed at the origin. Here, we solve Eq. 23 in the Laplace space to calculate the FPT of the particle to the absorbing boundary. To do that, we set $\tilde{Q}_{r}\left(s \mid x_{0}\right):=\int_{0}^{\infty} Q_{r}\left(t \mid x_{0}\right) e^{-s t} d t$ as the Laplace transform of 
$Q_{r}\left(t \mid x_{0}\right)$. Laplace transforming Eq. 23) we get

$$
\begin{aligned}
\frac{d^{2} \tilde{Q}_{r}\left(s \mid x_{0}\right)}{d x_{0}^{2}}-\left(\frac{\beta U_{0}}{x_{0}}\right) \frac{d \tilde{Q}_{r}\left(s \mid x_{0}\right)}{d x_{0}} & -\left(\frac{s+r}{D}\right) \tilde{Q}_{r}\left(s \mid x_{0}\right)= \\
& -\frac{1+r \tilde{Q}_{r}\left(s \mid x_{r}\right)}{D}, \quad \text { (D.5 }
\end{aligned}
$$

where we utilized the initial condition $Q_{r}\left(0 \mid x_{0}\right)=1$. In order to convert Eq. (D.54) to a homogeneous differential equation, we consider a shifted observable $\tilde{q}\left(s \mid x_{0}\right):=\tilde{Q}_{r}\left(s \mid x_{0}\right)-$ $\left[\left(1+r \tilde{Q}_{r}\left(s \mid x_{r}\right)\right) /(s+r)\right]$. Eq. D.54 in terms of $\tilde{q}\left(s \mid x_{0}\right)$ then reads

$$
\frac{d^{2} \tilde{q}\left(s \mid x_{0}\right)}{d x_{0}^{2}}-\left(\frac{\beta U_{0}}{x_{0}}\right) \frac{d \tilde{q}\left(s \mid x_{0}\right)}{d x_{0}}-\left(\frac{s+r}{D}\right) \tilde{q}\left(s \mid x_{0}\right)=0 .
$$

Next, we consider another change of variables, viz., $\tilde{q}\left(s \mid x_{0}\right)=$ $x_{0}^{v} \tilde{y}\left(s \mid x_{0}\right)$ with $v=\left(1+\beta U_{0}\right) / 2$. Eq. D.55 then reduces to

$$
x_{0}^{2} \frac{d^{2} \tilde{y}\left(s \mid x_{0}\right)}{d x_{0}^{2}}+x_{0} \frac{d \tilde{y}\left(s \mid x_{0}\right)}{d x_{0}}-\left(\alpha^{2} x_{0}^{2}+v^{2}\right) \tilde{y}\left(s \mid x_{0}\right)=0,
$$

where $\alpha=\sqrt{(s+r) / D}$. Eq. (D.56) is a modified Bessel equation with the general solution $\frac{87}{}$

$$
\tilde{y}\left(s \mid x_{0}\right)=A(s) I_{v}\left(\alpha x_{0}\right)+B(s) K_{v}\left(\alpha x_{0}\right),
$$

where $I_{v}\left(\alpha x_{0}\right)$ and $K_{v}\left(\alpha x_{0}\right)$ are modified Bessel functions of the first and second kind of order $v>0$, respectively. To obtain the specific solution of Eq. (D.56), we need to determine the explicit expressions of $A(s)$ and $B(s)$. We accomplish that in the following way.

We note that in the limit $x_{0} \rightarrow \infty$, i.e., when the initial position is very far from the absorbing boundary, chances are negligible that the particle is absorbed, and hence the survival probability is unity; $\left.Q_{r}\left(t \mid x_{0}\right)\right|_{x_{0} \rightarrow \infty}=1$. That leads to $\tilde{Q}_{r}(s \mid \infty)=1 / s$ and $\tilde{q}(s \mid \infty)=1 / s-\left[\left(1+r \tilde{Q}_{r}\left(s \mid x_{r}\right)\right) /(s+r)\right]$, which in turn gives $\tilde{y}(s \mid \infty)=0$. Since $K_{v}(\infty) \rightarrow 0$, but $I_{V}(\infty)$ diverges, we set $A(s)=0$ to keep things consistent.

To calculate $B(s)$, we first assume that the absorbing boundary is kept at $x=\varepsilon$. This leads to $\left.Q_{r}\left(t \mid x_{0}\right)\right|_{x_{0}=\varepsilon}=0$, since the survival probability vanishes if the process is initiated at the absorbing boundary. Therefore, $\tilde{Q}_{r}(s \mid \varepsilon)=0$ and $\tilde{q}(s \mid \varepsilon)=-\left[\left(1+r \tilde{Q}_{r}\left(s \mid x_{r}\right)\right) /(s+r)\right]$. Thus by definition $\tilde{y}(s \mid \varepsilon)=-\varepsilon^{-v}\left[\left(1+r \tilde{Q}_{r}\left(s \mid x_{r}\right)\right) /(s+r)\right] \equiv B(s) K_{v}(\alpha \varepsilon)$. Taking $\varepsilon \rightarrow 0$ to match the conditions of our problem, we have $K_{v}(\alpha \varepsilon) \simeq(\alpha \varepsilon)^{-v}\left[2^{v-1} \Gamma(v)\right]$, where $\Gamma(v)$ denotes the gamma function. Comparing, we get $B(s)=-\alpha^{v}(1+$ $\left.r \tilde{Q}_{r}\left(s \mid x_{r}\right)\right) /\left[2^{v-1} \Gamma(v)(s+r)\right]$. Thus the specific solution to Eq. D.56) reads

$$
\tilde{y}\left(s \mid x_{0}\right)=-\frac{1+r \tilde{Q}_{r}\left(s \mid x_{r}\right)}{2^{v-1} \Gamma(v)(s+r)} \alpha^{v} K_{v}\left(\alpha x_{0}\right) .
$$

Therefore, the specific solution to Eq. (D.54) is

$$
\tilde{Q}_{r}\left(s \mid x_{0}\right)=-\frac{1+r \tilde{Q}_{r}\left(s \mid x_{r}\right)}{s+r}\left[\frac{\left(\alpha x_{0}\right)^{v} K_{v}\left(\alpha x_{0}\right)}{2^{v-1} \Gamma(v)}-1\right] .
$$

We can evaluate $\tilde{Q}_{r}\left(s \mid x_{0}\right)$ in a self consistent manner by putting $x_{r}=x_{0}$ in the above equation, which means that the particle is taken back to the initial position after resetting. After simplification, this gives

$$
\tilde{Q}_{r}\left(s \mid x_{0}\right)=\frac{2^{v-1} \Gamma(v)-\left(\alpha x_{0}\right)^{v} K_{v}\left(\alpha x_{0}\right)}{s 2^{v-1} \Gamma(v)+r\left(\alpha x_{0}\right)^{v} K_{v}\left(\alpha x_{0}\right)},
$$

which is equivalent to Eq. (27) in the main text. Recalling that $f_{T_{r}}(t)=-d Q_{r}\left(t \mid x_{0}\right) / d t^{90}$, where $f_{T_{r}}(t)$ the probability density of $T_{r}$, we see that Eq. D.60) allows us to calculate any moment of $T_{r}$ following the relation

$$
\begin{aligned}
\left\langle T_{r}^{n}\right\rangle & =-\int_{0}^{\infty} t^{n}\left[\frac{\partial Q_{r}\left(t \mid x_{0}\right)}{\partial t}\right] d t \\
& =(-1)^{n-1} n\left[\frac{d^{n-1} \tilde{Q}_{r}\left(s \mid x_{0}\right)}{d s^{n-1}}\right]_{s=0} .
\end{aligned}
$$

In particular, the first moment or the mean FPT is given by $\left\langle T_{r}\right\rangle=\left[\tilde{Q}_{r}\left(s \mid x_{0}\right)\right]_{s=0}$. Indeed, setting $s=0$ in Eq. D.60), we recover Eq. 29] of the main text.

\section{APPENDIX E: LAPLACE TRANSFORM OF EQ. (5)}

Eq. (5) presents $f_{T}(t)$, the first passage time distribution to the origin for a particle that diffuses in a weakly repulsive or attractive logarithmic potential with $\beta U_{0}>-1$. Letting $\tilde{T}(s):=\int_{0}^{\infty} e^{-s t} f_{T}(t) d t$ denote the Laplace transform of $f_{T}(t)$, we utilize Eq. (5) to get

$$
\tilde{T}(s)=\frac{1}{\Gamma(v)}\left(\frac{x_{0}^{2}}{4 D}\right)^{v} \int_{0}^{\infty} t^{-(v+1)} \exp \left(-s t-\frac{x_{0}^{2}}{4 D t}\right) d t .
$$

Next, we note the following relation 92

$$
\int_{0}^{\infty} x^{\gamma-1} \exp \left(-x-\frac{\mu^{2}}{4 x}\right) d x=2\left(\frac{\mu}{2}\right)^{\gamma} K_{-\gamma}(\mu)
$$

which holds for $|\arg (\mu)|<\pi / 2$ and $\operatorname{Re}\left(\mu^{2}\right)>0$. Comparing the integrals in Eqs. (E.63) and (E.62) E.63, we identify $x \equiv s t, \gamma \equiv-v$ and $\mu \equiv \sqrt{s x_{0}^{2} / D}$. Evaluating the integral in Eq. (E.62) with the aid of Eq. (E.63) and plugging the result back into Eq. E.62), we obtain Eq. 25] in the main text.

\section{REFERENCES:}

${ }^{1}$ M. Luby, A. Sinclair, and D. Zuckerman, Optimal speedup of Las Vegas algorithms, Inf. Process Lett. 47, 173, (1993).

${ }^{2}$ C. P. Gomes, B. Selman, and H. Kautz, Boosting combinatorial search through randomization, AAAI/IAAI 98, 431, (1998).

${ }^{3}$ A. Montanari and R. Zecchina, Optimizing searches via rare events, Phys. Rev. Lett. 88, 178701, (2002).

${ }^{4}$ D. S. Steiger, T. F. Rønnow, and M. Troyer, Heavy tails in the distribution of time to solution for classical and quantum annealing, Phys. Rev. Lett. 115, 230501 (2015). 
${ }^{5} Ł$. Kuśmierz, S. N. Majumdar, S. Sabhapandit, and G. Schehr, First order transition for the optimal search time of Lévy flights with resetting, Phys. Rev. Lett. 113, 220602, (2014).

${ }^{6} €$. Kuśmierz, and E. Gudowska-Nowak, Optimal first-arrival times in Lévy flights with resetting, Phys. Rev. E 92, 052127, (2015).

${ }^{7} \mathrm{~S}$. Reuveni, Optimal stochastic restart renders fluctuations in first passage times universal, Phys. Rev. Lett. 116, 170601 (2016).

${ }^{8}$ A. Pal, A. Kundu, and M. R. Evans, Diffusion under time-dependent resetting, J. Phys. A: Math. Theor. 49 225001, (2016).

${ }^{9}$ U. Bhat, C. De Bacco, and S. Redner, Stochastic search with Poisson and deterministic resetting, J. Stat. Mech. 2016, 083401, (2016).

${ }^{10}$ A. Pal, and S. Reuveni, First Passage under Restart, Phys. Rev. Lett. 118, 030603, (2017).

${ }^{11} \mathrm{~A}$. Chechkin, and I. M. Sokolov, Random search with resetting: a unified renewal approach, Phys. Rev. Lett. 121, 050601, (2018).

${ }^{12}$ M. R. Evans, and S. N. Majumdar, Effects of refractory period on stochastic resetting, J. Phys. A: Math. Theor. 52, 01LT01, (2018).

${ }^{13}$ I. Eliazar, Branching search, EPL 120, 60008, (2018).

${ }^{14}$ A. Pal, I. Eliazar, and S. Reuveni, First passage under restart with branching, Phys. Rev. Lett. 122, 020602, (2019).

${ }^{15}$ G. M. Viswanathan, M. G. E. da Luz, E. P. Raposo, and H. E. Stanley, The Physics of Foraging: An Introduction to Random Searches and Biological Encounters, Cambridge U. Press, New York (2011).

${ }^{16} \mathrm{~A}$. Pal, L. Kuśmierz, and S. Reuveni, Home-range search provides advantage under high uncertainty, arXiv:1906.06987, (2019).

${ }^{17}$ P. Visco, R. J. Allen, S. N. Majumdar, and M. R. Evans, Switching and growth for microbial populations in catastrophic responsive environments, Biophys. J. 98, 1099 (2010).

${ }^{18}$ D. Sornette, Critical market crashes, Phys. Rep. 378, 1, (2003).

${ }^{19} \mathrm{~S}$. Reuveni, M. Urbakh, and J. Klafter, Role of substrate unbinding in MichaelisMenten enzymatic reactions, Proc. Natl. Acad. Sci. U. S. A. 111, 4391 (2014).

${ }^{20}$ T. Rotbart, S. Reuveni, and M. Urbakh, Michaelis-Menten reaction scheme as a unified approach towards the optimal restart problem, Phys. Rev. E $\mathbf{9 2}$, 060101 (2015).

${ }^{21}$ A. M. Berezhkovskii, A. Szabo, T. Rotbart, M. Urbakh, and A. B. Kolomeisky, Dependence of the Enzymatic Velocity on the Substrate Dissociation Rate, J. Phys. Chem. B 121, 3437 (2016).

${ }^{22}$ T. Robin, S. Reuveni, and M. Urbakh, Single-molecule theory of enzymatic inhibition, Nat. Commun. 9, 779 (2018).

${ }^{23}$ F. Wong, A. Dutta, D. Chowdhury, and J. Gunawardena, Structural conditions on complex networks for the Michaelis-Menten inputoutput response, Proc. Natl. Acad. Sci. USA 115, 9738, (2018).

${ }^{24}$ É. Roldán, A. Lisica, D. Sánchez-Taltavull, and S. W. Grill, Stochastic resetting in backtrack recovery by RNA polymerases, Phys. Rev. E 93, 062411 (2016).

${ }^{25}$ I. Eliazar, T. Koren, and J. Klafter, Searching circular DNA strands, J. Phys.: Condens. Matter 19, 065140 (2007).

${ }^{26}$ I. Eliazar, T. Koren, and J. Klafter, 2008. Parallel search of long circular strands: Modeling, analysis, and optimization, J. Phys. Chem. B 112, 5905, (2008).

${ }^{27}$ S. Budnar, K. B. Husain, G. A. Gomez, M. Naghibosadat, A. Varma, S. Verma, N. A. Hamilton, R. G. Morris, and A. S. Yap, Anillin promotes cell contractility by cyclic resetting of RhoA residence kinetics, Dev. Cell. 49, 894 (2019).

${ }^{28}$ Y. E. Kim, M. S. Hipp, A. Bracher, M. Hayer-Hartl, and F. U. Hartl, Molecular chaperone functions in protein folding and proteostasis, Annu. Rev. Biochem 82, 323 (2013).

${ }^{29}$ M. R. Evans, S. N. Majumdar, and G. Schehr, Stochastic resetting and applications, J. Phys. A: Math. Theor. in press, doi:10.1088/17518121/ab7cfe, (2020).

${ }^{30}$ M. R. Evans, and S. N. Majumdar, Diffusion with stochastic resetting, Phys. Rev. Lett. 106, 160601 (2011).

${ }^{31}$ M. R. Evans, and S. N. Majumdar, Diffusion with optimal resetting, J. Physics A: Math. Theor. 44, 435001 (2011).

${ }^{32}$ M. R. Evans, S. N. Majumdar, and K. Mallick, Optimal diffusive search: nonequilibrium resetting versus equilibrium dynamics, J. Physics A: Math. Theor. 46, 185001 (2013).

${ }^{33}$ M. R. Evans, and S. N. Majumdar, Diffusion with resetting in arbitrary spatial dimension, J. Physics A: Math. Theor. 47, 285001 (2014).
${ }^{34}$ C. Christou, and A. Schadschneider, Diffusion with resetting in bounded domains, J. Physics A: Math. Theor. 48, 285003 (2015).

${ }^{35} \mathrm{~A}$. Pal, A. Kundu, and M. R. Evans, Diffusion under time-dependent resetting, J. Physics A: Math. Theor. 49, 225001 (2016).

${ }^{36}$ A. Nagar, and S. Gupta, Diffusion with stochastic resetting at power-law times, Phys. Rev. E 93, 060102 (2016).

${ }^{37}$ S. Eule, and J. J. Metzger, Non-equilibrium steady states of stochastic processes with intermittent resetting, New J. Phys. 18, 033006 (2016).

${ }^{38} \mathrm{~A}$. Pal, and V. V. Prasad, First passage under stochastic resetting in an interval, Phys. Rev. E, 99, 032123 (2019).

${ }^{39}$ A. Pal, R. Chatterjee, S. Reuveni, and A. Kundu, Local time of diffusion with stochastic resetting, J. Physics A: Math. Theor. 52, 264002 (2019).

${ }^{40} \mathrm{~A}$. Pal, Ł. Kusmierz, and S. Reuveni, Time-dependent density of diffusion with stochastic resetting is invariant to return speed, Phys. Rev. E 100, 040101, (2019).

${ }^{41} \mathrm{~A}$. Pal, Ł. Kusmierz, and S. Reuveni, Invariants of motion with stochastic resetting and space-time coupled returns, New J. Phys. 21, 113024, (2019).

${ }^{42}$ O. Tal-Friedman, A. Pal, A. Sekhon, S. Reuveni, and Y. Roichman, Experimental realization of diffusion with stochastic resetting, arXiv:2003.03096, (2020).

${ }^{43} \mathrm{~S}$. Redner, A guide to first-passage processes, Cambridge University Press, (2001).

${ }^{44}$ A. J. Bray, S. N. Majumdar, and G. Schehr, Persistence and first-passage properties in nonequilibrium systems, Adv. Phys. 62, 225, (2013).

${ }^{45} \mathrm{~A}$. Pal, Diffusion in a potential landscape with stochastic resetting, Phys. Rev. E 91, 012113 (2015).

${ }^{46}$ A. Pal and V. V. Prasad, Landau theory of restart transitions, Phys. Rev. Research 1, 032001 (2019).

${ }^{47}$ S. Ray, D. Mondal, and S. Reuveni, Péclet number governs transition to acceleratory restart in drift-diffusion, J. Phys. A: Math. Theor. 52, 255002 (2019).

${ }^{48}$ S. Ahmad, I. Nayak, A. Bansal, A. Nandi, and D. Das, First passage of a particle in a potential under stochastic resetting: a vanishing transition of optimal resetting rate, Phys. Rev. E 99, 022130 (2019).

${ }^{49}$ É. Roldán and S. Gupta, Path-integral formalism for stochastic resetting: Exactly solved examples and shortcuts to confinement, Phys. Rev. E 96, 022130 (2017).

${ }^{50}$ D. Gupta, C. A. Plata, and A. Pal, Work Fluctuations and Jarzynski Equality in Stochastic Resetting, Phys. Rev. Lett. 124, 110608 (2020).

${ }^{51}$ D. Poland and H. A. Scheraga, Phase Transitions in One Dimension and the HelixCoil Transition in Polyamino Acids, J. Chem. Phys. 45, 1456 (1966).

${ }^{52}$ D. Poland and H. A. Scheraga, Occurrence of a Phase Transition in Nucleic Acid Models, J. Chem. Phys. 45, 1464 (1966).

${ }^{53}$ A. Bar, Y. Kafri, and D. Mukamel, Loop Dynamics in DNA Denaturation, Phys. Rev. Lett. 98, 038103 (2007).

${ }^{54}$ C. Fogedby and R. Metzler, DNA Bubble Dynamics as a Quantum Coulomb Problem, Phys. Rev. Lett. 98, 070601 (2007).

${ }^{55}$ A. Bar, Y. Kafri, and D. Mukamel, Dynamics of DNA melting, J. Phys.: Condens. Matter 21, 034110 (2009).

${ }^{56} \mathrm{~V}$. Kaiser and T. Novotný, Loop exponent in DNA bubble dynamics, J. Phys. A: Math. Theor. 47, 315003 (2014).

${ }^{57}$ D. A. Kessler and E. Barkai, Infinite Covariant Density for Diffusion in Logarithmic Potentials and Optical Lattices, Phys. Rev. Lett. 105, 120602 (2010).

${ }^{58}$ A. Dechant, E. Lutz, D. A. Kessler, and E. Barkai, Fluctuations of Time Averages for Langevin Dynamics in a Binding Force Field, Phys. Rev. Lett. 107, 240603 (2011).

${ }^{59}$ A. Dechant, E. Lutz, E. Barkai, and D. A. Kessler, Solution of the FokkerPlanck Equation with a Logarithmic Potential, J. Stat. Phys. 145, 1524 (2011).

${ }^{60}$ A. Dechant, E. Lutz, D. A. Kessler, and E. Barkai, Superaging correlation function and ergodicity breaking for Brownian motion in logarithmic potentials, Phys. Rev. E 85, 051124 (2012).

${ }^{61}$ D. A. Kessler and E. Barkai, Theory of Fractional Lévy Kinetics for Cold Atoms Diffusing in Optical Lattices, Phys. Rev. Lett. 108, 230602 (2012).

${ }^{62} \mathrm{E}$. Lutz and F. Renzoni, Beyond Boltzmann-Gibbs statistical mechanics in optical lattices, Nat. Phys. 9, 615 (2013).

${ }^{63}$ N. Leibovich and E. Barkai, Aging Wiener-Khinchin Theorem, Phys. Rev. Lett. 115, 080602 (2015). 
${ }^{64}$ N. Leibovich, A. Dechant, E. Lutz, and E. Barkai, Aging Wiener-Khinchin Theorem and and critical exponents of $1 / f^{\beta}$ noise, Phys. Rev. E 94, 052130 (2016).

${ }^{65}$ R. Zwanzig, Diffusion Past an Entropy Barrier, J. Phys. Chem. 96, 3926 (1992).

${ }^{66}$ D. Reguera and J. M. Rubí, Kinetic equations for diffusion in the presence of entropic barriers, Phys. Rev. E 64, 061106 (2001).

${ }^{67}$ M. Muthukumar, Polymer escape through a nanopore, J. Chem. Phys. 118, 5174 (2003)

${ }^{68}$ D. Mondal, M. Das, and D. S. Ray, Entropic resonant activation, J. Chem. Phys. 132, 224102 (2010).

${ }^{69}$ D. Mondal, M. Das, and D. S. Ray, Entropic noise-induced nonequilibrium transition, J. Chem. Phys. 133, 204102 (2010).

${ }^{70}$ D. Mondal, Enhancement of entropic transport by intermediates, Phys. Rev. E 84, 011149 (2011).

${ }^{71}$ D. Mondal, and D. S. Ray, Asymmetric stochastic localization in geometry controlled kinetics, J. Chem. Phys. 135, 194111 (2011).

${ }^{72}$ F. J. Dyson, A Brownian-Motion Model for the Eigenvalues of a Random Matrix, J. Math. Phys. 3, 1191 (1962).

${ }^{73} \mathrm{H}$. Spohn, Tracer dynamics in Dyson's model of interacting Brownian particles, J. Stat. Phys. 47, 669 (1987).

${ }^{74} \mathrm{~F}$. Bouchet and T. Dauxois, Prediction of anomalous diffusion and algebraic relaxations for long-range interacting systems, using classical statistical mechanics, Phys. Rev. E 72, 045103 (1992).

${ }^{75}$ P. H. Chavanis, C. Rosier, and C. Sire, Thermodynamics of self-gravitating systems, Phys. Rev. E 66, 036105 (2002).

${ }^{76}$ C. Sire and P. H. Chavanis, Thermodynamics and collapse of selfgravitating Brownian particles in D dimensions, Phys. Rev. E 66, 046133 (2002).

${ }^{77}$ S. Manning, Limiting Laws and Counterion Condensation in Polyelectrolyte Solutions I. Colligative Properties, J. Chem. Phys. 51, 924 (1969).

${ }^{78} \mathrm{D}$. Mondal and M. Muthukumar Ratchet rectification effect on the translocation of a flexible polyelectrolyte chain, J. Chem. Phys. 145, 084906 (2016).
${ }^{79}$ E. Levine, D. Mukamel, and G. M. Schütz, Zero-Range Process with Open Boundaries, EPL 47, 565 (2005).

${ }^{80}$ O. Hirschberg, D. Mukamel and G. M. Schütz, Approach to equilibrium of diffusion in a logarithmic potential, Phys. Rev. E 84, 041111 (2011).

${ }^{81}$ O. Hirschberg, D. Mukamel and G. M. Schütz, Diffusion in a logarithmic potential: scaling and selection in the approach to equilibrium, J. Stat. Mech. 2012, 02001 (2012).

${ }^{82} \mathrm{~A}$. J. Bray, Random walks in logarithmic and power-law potentials, nonuniversal persistence, and vortex dynamics in the two-dimensional XY model, Phys. Rev. E 62, 103 (2000).

${ }^{83}$ E. Martin, U. Behn and G. Germano, First-passage and first-exit times of a Bessel-like stochastic process, Phys. Rev. E 83, 051115 (2011).

${ }^{84}$ A. Ryabov, E. Berestneva, and V. Holubec, Brownian motion in timedependent logarithmic potential: Exact results for dynamics and firstpassage properties, J. Chem. Phys. 143, 114117 (2015).

${ }^{85}$ D. R. Cox, and H.D. Miller, The Theory of Stochastic Processes, CRC Press, (2001) [See Eq. (74) in Chapter 5].

${ }^{86}$ Digital Library of Mathematical Functions, National Institute of Standards and Technology (NIST), U.S. Department of Commerce, https://dlmf.nist.gov/.

${ }^{87}$ M. Abramowitz and I. A. Stegun, Handbook of Mathematical Functions: with Formulas, Graphs, and Mathematical Tables, National Bureau of Standards, (1970) [See Eq. 9.6.1 in page 374].

${ }^{88}$ A. Erdélyi, W. Magnus, F. Oberhettinger and F. G. Tricomi, Tables of Integral Transforms, Volume I, California Institute of Technology, Bateman Manuscript Project (Based, in part, on notes left by Late Prof. Harry Batemann), McGrawHill Book Comp., Inc. (1954). [See Eq. (4) in page 200].

${ }^{89}$ A. Laforgia and P. Natalini, Some Inequalities for Modified Bessel Functions, J. Inequal. Appl. 2010, 253035 (2010).

${ }^{90}$ C. W. Gardiner, Handbook of Stochastic Methods for Physics, Chemistry and the Natural Sciences, Springer-Verlag., (2003).

${ }^{91}$ C. Xavier, Fortran 77 and Numerical Methods, New Age International, (1994).

${ }^{92}$ I. S. Gradshtein, I. M. Ryzhik, D. Zwillinger and V. Moll, Table of integrals, series, and products, Academic Press, Elsevier Inc., 8th Edition, (2014). [See Eq. 12 of Section 3.474 in page 371]. 